Noname manuscript No.

(will be inserted by the editor)

\title{
Geometric and Metric Characterizations of Transversality Properties
}

\author{
Hoa T. Bui · Nguyen Duy Cuong · Alexander Y. \\ Kruger \\ Dedicated to Professor Marco Antonio López Cerdá on the occasion of his $70^{\text {th }}$ birthday
}

Received: date / Accepted: date

\begin{abstract}
This paper continues the study of 'good arrangements' of collections of sets near a point in their intersection. We clarify quantitative relations between several geometric and metric characterizations of the transversality properties of collections of sets and the corresponding regularity properties of set-valued mappings. We expose all the parameters involved in the definitions and characterizations and establish relations between them. This allows us to classify the quantitative geometric and metric characterizations of transversality and regularity, and subdivide them into two groups with complete exact equivalences between the parameters within each group and clear relations between the values of the parameters in different groups.
\end{abstract}

Keywords Transversality - Subtransversality - Semitransversality · Regularity · Subregularity $\cdot$ Semiregularity

Mathematics Subject Classification (2000) 49J52 - 49J53 • 49K40 • 90C30 - 90C46

\section{Introduction}

We continue studying 'good arrangements' of collections of sets in normed vector spaces near a point in their intersection, known as transversality (regularity) properties and playing an important role in optimization and variational analysis, e.g., as constraint qualifications in optimality conditions, and qualification conditions in subdifferential, normal cone and coderivative calculus, and convergence analysis of computational algorithms $[2-6,9,10,12,13,15,17,19-32,34-38,40,41]$.

Our aim in this paper is to clarify relations between several quantitative geometric and metric characterizations of the transversality properties of collections of sets and the corresponding regularity properties of set-valued mappings. We study here three typical properties of collections of sets: $\alpha$-semitransversality, $\alpha$-subtransversality and $\alpha$-transversality and the corresponding three properties of set-valued mappings: $\alpha$-semiregularity, $\alpha$-subregularity and $\alpha$-regularity; see Definitions 1 and 2 .

The first two properties in each group admit unique quantitative metric characterizations; see Theorems 2 and 3. The situation with the remaining pair of properties is more complicated: they admit several metric characterizations, which are not entirely equivalent quantitatively: they differ in the values of certain auxiliary parameters. This has been a source of some confusion in the recent literature and added unnecessary complications, e.g., in the statements and proofs of the quantitative relations between transversality and regularity properties; cf. [29, Theorem 5.1].

Hoa T. Bui · Nguyen Duy Cuong · Alexander Y. Kruger ( $\varangle)$

Centre for Informatics and Applied Optimization, School of Science, Engineering and Information Technology, Federation University Australia, POB 663, Ballarat, Vic, 3350, Australia

E-mail: h.bui@federation.edu.au,duynguyen@students.federation.edu.au, a.kruger@federation.edu.au 
In this paper, we expose all the parameters involved in the definitions and characterizations and establish relations between them. This allows us to classify all quantitative geometric and metric characterizations of $\alpha$-transversality properties and subdivide them into two groups with complete exact equivalencies between the parameters within each group and clear relations between the values of the parameters in different groups; cf. Propositions 5 and 9. As a by-product, we establish straightforward two-way quantitative relations between the transversality properties of collections of sets and the corresponding regularity properties of set-valued mappings, improving and streamlining the corresponding results from [29].

To simplify the presentation, only 'linear' transversality and regularity properties are considered here. For some recent nonlinear extensions we refer the readers to [11-13]. The convex case is given a special attention.

The rest of the paper is organized as follows. In Section 2, we recall conventional definitions of the transversality properties of a collection of arbitrary subsets of a normed vector space, having a common point, and discuss basic relations between them. In Section 3 , we establish equivalent geometric characterizations of $\alpha$-subtransversality and $\alpha$-transversality. Section 4 is dedicated to metric characterizations of the transversality properties. In Section 5, we clarify quantitative relations between the transversality properties of collections of sets and the corresponding regularity properties of set-valued mappings.

Our basic notation is standard, see, e.g., $[14,33,39]$. The open unit balls in normed vector spaces $X$ and $Y$ are denoted by $\mathbb{B}_{X}$ and $\mathbb{B}_{Y}$, respectively. When it does not cause confusion, we simply write $\mathbb{B}$. The open ball with center $x$ and radius $\delta>0$ is denoted by $B_{\delta}(x)$. If not explicitly stated otherwise, products of normed spaces are assumed to be equipped with the maximum norm $\|(x, y)\|:=\max \{\|x\|,\|y\|\},(x, y) \in X \times Y$.

For a set $\Omega \subset X$, its interior and boundary are denoted by int $\Omega$ and bd $\Omega$, respectively. The distance from a point $x \in X$ to a set $\Omega \subset X$ is defined by $d(x, \Omega):=\inf _{u \in \Omega}\|u-x\|$, and we use the convention $d(x, \emptyset)=+\infty$.

A set-valued mapping $F: X \rightrightarrows Y$ between two sets $X$ and $Y$ is a mapping, which assigns to every $x \in X$ a subset (possibly empty) $F(x)$ of $Y$. We use the notations gph $F:=$ $\{(x, y) \in X \times Y: y \in F(x)\}$ and $\operatorname{dom} F:=\{x \in X: F(x) \neq \emptyset\}$ for the graph and the domain of $F$, respectively, and $F^{-1}: Y \rightrightarrows X$ for the inverse of $F$. This inverse (which always exists with possibly empty values at some $y$ ) is defined by $F^{-1}(y):=\{x \in X: y \in F(x)\}, y \in Y$. Obviously $\operatorname{dom} F^{-1}=F(X)$.

\section{Definitions and Basic Relations}

In this section, we recall conventional definitions of the transversality properties of a collection of $n \geq 2$ arbitrary subsets $\Omega_{1}, \ldots, \Omega_{n}$ of a normed vector space, having a common point, and discuss basic relations between them. We write $\left\{\Omega_{1}, \ldots, \Omega_{n}\right\}$ to denote the collection of sets as a single object.

The next definition is a modification of [29, Definition 3.1].

Definition 1 Let $\Omega_{1}, \ldots, \Omega_{n}$ be subsets of a normed space $X, \bar{x} \in \cap_{i=1}^{n} \Omega_{i}$, and $\alpha>0$. The collection $\left\{\Omega_{1}, \ldots, \Omega_{n}\right\}$ is

(i) $\alpha$-semitransversal at $\bar{x}$ if there exists a $\delta>0$ such that

$$
\bigcap_{i=1}^{n}\left(\Omega_{i}-x_{i}\right) \cap B_{\rho}(\bar{x}) \neq \emptyset
$$

for all $\rho \in] 0, \delta\left[\right.$ and $x_{i} \in X(i=1, \ldots, n)$ with $\max _{1 \leq i \leq n}\left\|x_{i}\right\|<\alpha \rho$;

(ii) $\alpha$-subtransversal at $\bar{x}$ if there exist $\delta_{1}>0$ and $\delta_{2}>0$ such that

$$
\bigcap_{i=1}^{n} \Omega_{i} \cap B_{\rho}(x) \neq \emptyset
$$

for all $\rho \in] 0, \delta_{1}\left[\right.$ and $x \in B_{\delta_{2}}(\bar{x})$ with $\max _{1 \leq i \leq n} d\left(x, \Omega_{i}\right)<\alpha \rho$; 
(iii) $\alpha$-transversal at $\bar{x}$ if there exist $\delta_{1}>0$ and $\delta_{2}>0$ such that

$$
\bigcap_{i=1}^{n}\left(\Omega_{i}-\omega_{i}-x_{i}\right) \cap(\rho \mathbb{B}) \neq \emptyset
$$

for all $\rho \in] 0, \delta_{1}\left[, \omega_{i} \in \Omega_{i} \cap B_{\delta_{2}}(\bar{x})\right.$ and $x_{i} \in X(i=1, \ldots, n)$ with $\max _{1 \leq i \leq n}\left\|x_{i}\right\|<\alpha \rho$.

The definition of $\alpha$-subtransversality in part (ii) differs from the corresponding one in [29, Definition 3.1]. In view of Theorem 1, the two representations are equivalent.

Each of the properties in Definition 1 is determined by a number $\alpha>0$, playing the role of a rate of the respective property, and either a number $\delta>0$ in item (i) or two numbers $\delta_{1}>0$ and $\delta_{2}>0$ in items (ii) and (iii). The exact upper bound of all $\alpha>0$ such that the property holds with some $\delta>0$, or $\delta_{1}>0$ and $\delta_{2}>0$, is called the modulus of this property. We use the notations $\operatorname{se} \operatorname{tr}\left[\Omega_{1}, \ldots, \Omega_{n}\right](\bar{x}), \operatorname{str}\left[\Omega_{1}, \ldots, \Omega_{n}\right](\bar{x})$ and $\operatorname{tr}\left[\Omega_{1}, \ldots, \Omega_{n}\right](\bar{x})$ for the moduli of the respective properties. If the property does not hold, then by convention the respective modulus equals 0 .

If $\left\{\Omega_{1}, \ldots, \Omega_{n}\right\}$ is $\alpha$-semitransversal (respectively, $\alpha$-subtransversal or $\alpha$-transversal) at $\bar{x}$ with some $\alpha>0$ and $\delta>0$ (respectively, $\delta_{1}>0$ and $\delta_{2}>0$ ), we often simply say that $\left\{\Omega_{1}, \ldots, \Omega_{n}\right\}$ is semitransversal (respectively, subtransversal or transversal) at $\bar{x}$.

The role of the $\delta$ 's in the definitions is more technical: they control the size of the interval for the values of $\rho$ and, in the case of subtransversality and transversality in parts (ii) and (iii), the size of the neighbourhoods of $\bar{x}$ involved in the respective definitions. Of course, if a property is satisfied with some $\delta_{1}>0$ and $\delta_{2}>0$, it is also satisfied with the single $\delta:=\min \left\{\delta_{1}, \delta_{2}\right\}$ in place of both $\delta_{1}$ and $\delta_{2}$. We use here two different parameters to emphasise their different roles in the definitions and the corresponding characterizations. Moreover, we are going to provide quantitative estimates for the values of these parameters, which can be important in applications.

If all the sets coincide, the subtransversality property is satisfied trivially.

Proposition 1 Let $\Omega$ be a subset of a normed space $X, \bar{x} \in \Omega$, and $\alpha \in] 0,1]$. The collection $\{\Omega, \ldots, \Omega\}$ of $n \geq 2$ copies of $\Omega$ is $\alpha$-subtransversal at $\bar{x}$.

Proof Observe that condition (2) in the current setting takes the form $\Omega \cap B_{\rho}(x) \neq \emptyset$, and is trivially satisfied if $d(x, \Omega)<\alpha \rho$.

The next result shows that $\alpha$-transversality is the strongest of the three properties in Definition 1.

Proposition 2 Let $\Omega_{1}, \ldots, \Omega_{n}$ be subsets of a normed space $X, \bar{x} \in \cap_{i=1}^{n} \Omega_{i}$, and $\alpha>0$. If $\left\{\Omega_{1}, \ldots, \Omega_{n}\right\}$ is $\alpha$-transversal at $\bar{x}$ with some $\delta_{1}>0$ and $\delta_{2}>0$, then it is $\alpha$-semitransversal at $\bar{x}$ with $\delta:=\delta_{1}$ and $\alpha$-subtransversal at $\bar{x}$ with any $\left.\left.\delta_{1}^{\prime} \in\right] 0, \delta_{1}\right]$ and $\delta_{2}^{\prime}>0$ such that $\alpha \delta_{1}^{\prime}+\delta_{2}^{\prime} \leq \delta_{2}$.

As a consequence, $\mathrm{s}_{\mathrm{e}} \operatorname{tr}\left[\Omega_{1}, \ldots, \Omega_{n}\right](\bar{x}) \geq \operatorname{tr}\left[\Omega_{1}, \ldots, \Omega_{n}\right](\bar{x})$ and $\operatorname{str}\left[\Omega_{1}, \ldots, \Omega_{n}\right](\bar{x}) \geq$ $\operatorname{tr}\left[\Omega_{1}, \ldots, \Omega_{n}\right](\bar{x})$.

Proof Suppose $\left\{\Omega_{1}, \ldots, \Omega_{n}\right\}$ is $\alpha$-transversal at $\bar{x}$ with some $\delta_{1}>0$ and $\delta_{2}>0$. Since condition (1) is a particular case of condition (3) with $\omega_{i}=\bar{x}(i=1, \ldots, n)$, we conclude that $\left\{\Omega_{1}, \ldots, \Omega_{n}\right\}$ is $\alpha$-semitransversal at $\bar{x}$ with $\delta:=\delta_{1}$. Let $\left.\left.\delta_{1}^{\prime} \in\right] 0, \delta_{1}\right]$ and $\delta_{2}^{\prime}>0$ be such that $\alpha \delta_{1}^{\prime}+\delta_{2}^{\prime} \leq \delta_{2}$, and let $\left.\rho \in\right] 0, \delta_{1}^{\prime}\left[\right.$ and $x \in B_{\delta_{2}^{\prime}}(\bar{x})$ with $\max _{1 \leq i \leq n} d\left(x, \Omega_{i}\right)<\alpha \rho$. Choose $\omega_{i} \in \Omega_{i}(i=1, \ldots, n)$ such that $\max _{1 \leq i \leq n}\left\|x-\omega_{i}\right\|<\alpha \rho$. Then

$$
\left\|\omega_{i}-\bar{x}\right\| \leq\left\|x-\omega_{i}\right\|+\|x\|<\alpha \rho+\delta_{2}^{\prime}<\delta_{2}(i=1, \ldots, n) .
$$

Set $x_{i}:=x-\omega_{i}(i=1, \ldots, n)$. We have $\left.\rho \in\right] 0, \delta_{1}\left[, \omega_{i} \in \Omega_{i} \cap B_{\delta_{2}}(\bar{x})(i=1, \ldots, n)\right.$ and $\max _{1 \leq i \leq n}\left\|x_{i}\right\|<\alpha \rho$. By Definition 1(iii), condition (3) is satisfied. This is equivalent to condition (2). In view of Definition 1(ii), $\left\{\Omega_{1}, \ldots, \Omega_{n}\right\}$ is $\alpha$-subtransversal at $\bar{x}$ with $\delta_{1}^{\prime}$ and $\delta_{2}^{\prime}$. 
Remark 1 Note that $\alpha$-semitransversality and $\alpha$-subtransversality are in general incomparable; cf. [29, Section 3.2].

The three transversality properties in Definition 1 are only meaningful when $\bar{x} \in \mathrm{bd} \cap \cap_{i=1}^{n} \Omega_{i}$.

Proposition 3 Let $\Omega_{1}, \ldots, \Omega_{n}$ be subsets of a normed space $X$. If $\bar{x} \in \operatorname{int} \cap_{i=1}^{n} \Omega_{i}$, then, for any $\alpha>0$, all three properties in Definition 1 hold true.

Proof Let $\bar{x} \in$ int $\cap_{i=1}^{n} \Omega_{i}$ and $\alpha>0$. In view of Proposition 2, we only need to prove that $\left\{\Omega_{1}, \ldots, \Omega_{n}\right\}$ is $\alpha$-transversal at $\bar{x}$. Choose numbers $\delta_{1}>0$ and $\delta_{2}>0$ such that, with $\delta:=\alpha \delta_{1}+\delta_{2}$, it holds $B_{\delta}(\bar{x}) \subset \cap_{i=1}^{n} \Omega_{i}$. Then, for all $\omega_{i} \in \Omega_{i} \cap B_{\delta_{2}}(\bar{x})$ and $x_{i} \in X$ $(i=1, \ldots, n)$ with $\max _{1 \leq i \leq n}\left\|x_{i}\right\|<\alpha \delta_{1}$, it holds $0 \in \cap_{i=1}^{n}\left(\Omega_{i}-\omega_{i}-x_{i}\right)$, and consequently, condition (3) is satisfied with any $\rho>0$. By Definition 1 (iii), $\left\{\Omega_{1}, \ldots, \Omega_{n}\right\}$ is $\alpha$-transversal at $\bar{x}$ with $\delta_{1}$ and $\delta_{2}$.

If $\cap_{i=1}^{n} \Omega_{n}$ is closed and $\bar{x} \in$ bd $\cap_{i=1}^{n} \Omega_{i}$, then $\alpha$-subtransversality and $\alpha$-transversality can only hold with $\alpha \leq 1$.

Proposition 4 Let $\Omega_{1}, \ldots, \Omega_{n}$ be subsets of a normed space $X, \cap_{i=1}^{n} \Omega_{n}$ be closed, $\bar{x} \in$ bd $\cap_{i=1}^{n} \Omega_{i}$, and $\alpha>0$. If $\left\{\Omega_{1}, \ldots, \Omega_{n}\right\}$ is $\alpha$-subtransversal (in particular, $\alpha$-transversal) at $\bar{x}$, then $\alpha \leq 1$.

Proof Let $\left\{\Omega_{1}, \ldots, \Omega_{n}\right\}$ be $\alpha$-subtransversal at $\bar{x}$ with some $\delta_{1}>0$ and $\delta_{2}>0$. Choose a point $x \in B_{\min }\left\{\alpha \delta_{1}, \delta_{2}\right\}(\bar{x}) \backslash \cap_{i=1}^{n} \Omega_{i}$. Then

$$
0<\max _{1 \leq i \leq n} d\left(x, \Omega_{i}\right) \leq d\left(x, \cap_{i=1}^{n} \Omega_{i}\right)<\alpha \delta_{1},
$$

and by Definition 1(ii), $\cap_{i=1}^{n} \Omega_{i} \cap B_{\rho}(x) \neq \emptyset$, i.e. $d\left(x, \cap_{i=1}^{n} \Omega_{i}\right)<\rho$, for all $\left.\rho \in\right] 0, \delta_{1}[$ satisfying $d\left(x, \cap_{i=1}^{n} \Omega_{i}\right)<\alpha \rho$, which is only possible when $\alpha \leq 1$.

Remark 2 With the exception of Proposition 4, we usually do not need the closedness of the sets when formulating equivalent geometric and metric characterizations of the three transversality properties as well as establishing their relations with the regularity properties of set-valued mappings. This assumption is essential when establishing primal (slope) and dual sufficient conditions for the properties; cf. [11, 28, 29].

\section{Geometric Characterizations}

The next statement provides two equivalent geometric characterizations of $\alpha$-subtransversality, which can serve as equivalent definitions of the property.

Theorem 1 Let $\Omega_{1}, \ldots, \Omega_{n}$ be subsets of a normed space $X, \bar{x} \in \cap_{i=1}^{n} \Omega_{i}$, and $\alpha>0$. The collection $\left\{\Omega_{1}, \ldots, \Omega_{n}\right\}$ is $\alpha$-subtransversal at $\bar{x}$ with some $\delta_{1}>0$ and $\delta_{2}>0$ if and only if the following equivalent conditions hold:

(i) for all $\rho \in] 0, \delta_{1}[$, it holds

$$
\bigcap_{i=1}^{n}\left(\Omega_{i}+(\alpha \rho) \mathbb{B}\right) \cap B_{\delta_{2}}(\bar{x}) \subset \bigcap_{i=1}^{n} \Omega_{i}+\rho \mathbb{B} ;
$$

(ii) condition (3) is satisfied for all $\rho \in] 0, \delta_{1}\left[, \omega_{i} \in \Omega_{i}\right.$ and $x_{i} \in X(i=1, \ldots, n)$ with $\max _{1 \leq i \leq n}\left\|x_{i}\right\|<\alpha \rho$ and $\omega_{1}+x_{1}=\ldots=\omega_{n}+x_{n} \in B_{\delta_{2}}(\bar{x})$. 
Proof Observe that condition (4) is equivalent to the inclusion $x \in \cap_{i=1}^{n} \Omega_{i}+\rho \mathbb{B}$ holding for all $x \in B_{\delta_{2}}(\bar{x})$ with $\max _{1 \leq i \leq n} d\left(x, \Omega_{i}\right)<\alpha \rho$. In its turn, this inclusion is equivalent to condition (2). This observation proves the equivalent characterization of $\alpha$-subtransversality in (a).

To prove the second equivalence, it is sufficient to notice that condition (2) can be rewritten as $\cap_{i=1}^{n}\left(\Omega_{i}-x\right) \cap(\rho \mathbb{B}) \neq \emptyset$, while the inequalities $\max _{1 \leq i \leq n} d\left(x, \Omega_{i}\right)<\alpha \rho$ are equivalent to the representations $x=\omega_{1}+x_{1}=\ldots=\omega_{n}+x_{n}$, where $\omega_{i} \in \Omega_{i}(i=1, \ldots, n)$ and $\max _{1 \leq i \leq n}\left\|x_{i}\right\|<\alpha \rho$. With such $x_{i}$ and $\omega_{i}(i=1, \ldots, n)$, the above condition is equivalent to condition (3).

The transversality property in part (iii) of Definition 1 admits several alternative representations. The three equivalent representations in the next proposition are of independent interest. They differ from the one in Definition 1(iii) by values of the parameters $\delta_{1}$ and $\delta_{2}$. The relations between the values of parameters in the two groups of representations can be estimated.

Proposition 5 Let $\Omega_{1}, \ldots, \Omega_{n}$ be subsets of a normed space $X, \bar{x} \in \cap_{i=1}^{n} \Omega_{i}, \alpha>0, \delta_{1}>0$ and $\delta_{2}>0$. The following conditions are equivalent:

(i) condition (3) holds for all $\rho \in] 0, \delta_{1}\left[, \omega_{i} \in \Omega_{i}\right.$ and $x_{i} \in X$ with $\omega_{i}+x_{i} \in B_{\delta_{2}}(\bar{x})$ $(i=1, \ldots, n)$ and $\max _{1 \leq i \leq n}\left\|x_{i}\right\|<\alpha \rho$;

(ii) condition (1) holds for all $\rho \in] 0, \delta_{1}\left[\right.$ and $x_{i} \in \delta_{2} \mathbb{B}(i=1, \ldots, n)$ with $\max _{1 \leq i \leq n} d\left(\bar{x}, \Omega_{i}-\right.$ $\left.x_{i}\right)<\alpha \rho$;

(iii) for all $\rho \in] 0, \delta_{1}\left[\right.$ and $x, x_{i} \in X$ with $x+x_{i} \in B_{\delta_{2}}(\bar{x}) \quad(i=1, \ldots, n)$ and $\max _{1 \leq i \leq n} d\left(x, \Omega_{i}-x_{i}\right)<\alpha \rho$, it holds

$$
\bigcap_{i=1}^{n}\left(\Omega_{i}-x_{i}\right) \cap B_{\rho}(x) \neq \emptyset .
$$

Moreover, if $\left\{\Omega_{1}, \ldots, \Omega_{n}\right\}$ is $\alpha$-transversal at $\bar{x}$ with some $\delta_{1}>0$ and $\delta_{2}>0$, then conditions (i)-(iii) hold with any $\left.\left.\delta_{1}^{\prime} \in\right] 0, \delta_{1}\right]$ and $\delta_{2}^{\prime}>0$ satisfying $\alpha \delta_{1}^{\prime}+\delta_{2}^{\prime} \leq \delta_{2}$ in place of $\delta_{1}$ and $\delta_{2}$.

Conversely, if conditions (i)-(iii) hold with some $\delta_{1}>0$ and $\delta_{2}>0$, then $\left\{\Omega_{1}, \ldots, \Omega_{n}\right\}$ is $\alpha$-transversal at $\bar{x}$ with any $\left.\left.\delta_{1}^{\prime} \in\right] 0, \delta_{1}\right]$ and $\delta_{2}^{\prime}>0$ satisfying $\alpha \delta_{1}^{\prime}+\delta_{2}^{\prime} \leq \delta_{2}$.

Proof We first prove the equivalence of conditions (i)-(iii).

(iii) $\Rightarrow$ (ii). This implication is trivial.

(ii) $\Rightarrow$ (i). Let $\rho \in] 0, \delta_{1}\left[, \omega_{i} \in \Omega_{i}, x_{i} \in X\right.$ with $\omega_{i}+x_{i} \in B_{\delta_{2}}(\bar{x})(i=1, \ldots, n)$ and $\max _{1 \leq i \leq n}\left\|x_{i}\right\|<\alpha \rho$. Set $x_{i}^{\prime}:=x_{i}+\omega_{i}-\bar{x}(i=1, \ldots, n)$. Then $x_{i}^{\prime} \in \delta_{2} \mathbb{B}$ and $d\left(\bar{x}, \Omega_{i}-x_{i}^{\prime}\right) \leq$ $\left\|\bar{x}-\omega_{i}+x_{i}^{\prime}\right\|=\left\|x_{i}\right\|<\alpha \rho(i=1, \ldots, n)$. By (ii), condition (1) is satisfied with $x_{i}^{\prime}$ in place of $x_{i}(i=1, \ldots, n)$. This is equivalent to condition (3).

(i) $\Rightarrow$ (iii). Let $\rho \in] 0, \delta_{1}\left[, x, x_{i} \in X\right.$ with $x+x_{i} \in B_{\delta_{2}}(\bar{x})(i=1, \ldots, n)$ and $\max _{1 \leq i \leq n} d\left(x, \Omega_{i}-x_{i}\right)<\alpha \rho$. Choose $\omega_{i} \in \Omega_{i}$ such that $\left\|x-\omega_{i}+x_{i}\right\|<\alpha \rho$ and set $x_{i}^{\prime}:=x-\omega_{i}+x_{i}(i=1, \ldots, n)$. We have $\omega_{i}+x_{i}^{\prime}=x+x_{i} \in B_{\delta_{2}}(\bar{x})$ and $\left\|x_{i}^{\prime}\right\|<\alpha \rho$ $(i=1, \ldots, n)$. In view of (i), condition (3) is satisfied with $x_{i}^{\prime}$ in place of $x_{i}(i=1, \ldots, n)$. This is equivalent to condition (5).

Suppose $\left\{\Omega_{1}, \ldots, \Omega_{n}\right\}$ is $\alpha$-transversal at $\bar{x}$ with some $\delta_{1}>0$ and $\delta_{2}>0$, and let $\left.\left.\delta_{1}^{\prime} \in\right] 0, \delta_{1}\right]$ and $\delta_{2}^{\prime}>0$ be such that $\alpha \delta_{1}^{\prime}+\delta_{2}^{\prime} \leq \delta_{2}$. Then, for all $\left.\rho \in\right] 0, \delta_{1}^{\prime}[$, $\omega_{i} \in \Omega_{i}, x_{i} \in X$ with $\omega_{i}+x_{i} \in B_{\delta_{2}^{\prime}}(\bar{x})(i=1, \ldots, n)$ and $\max _{1 \leq i \leq n}\left\|x_{i}\right\|<\alpha \rho$, we have $\left\|\omega_{i}-\bar{x}\right\| \leq\left\|x_{i}\right\|+\left\|\omega_{i}+x_{i}-\bar{x}\right\|<\alpha \delta_{1}^{\prime}+\delta_{2}^{\prime} \leq \delta_{2}(i=1, \ldots, n)$. By Definition 1(iii), condition (3) is satisfied, and consequently, condition (i) (as well as conditions (ii) and (iii)) holds with $\delta_{1}^{\prime}$ and $\delta_{2}^{\prime}$.

Conversely, suppose conditions (i)-(iii) hold with some $\delta_{1}>0$ and $\delta_{2}>0$, and let $\delta_{1}^{\prime} \in$ ] $\left.0, \delta_{1}\right]$ and $\delta_{2}^{\prime}>0$ be such that $\alpha \delta_{1}^{\prime}+\delta_{2}^{\prime} \leq \delta_{2}$. Then, for all $\left.\rho \in\right] 0, \delta_{1}^{\prime}\left[, \omega_{i} \in \Omega_{i} \cap B_{\delta_{2}^{\prime}}(\bar{x})\right.$ and $x_{i} \in X(i=1, \ldots, n)$ with $\max _{1 \leq i \leq n}\left\|x_{i}\right\|<\alpha \rho$, we have $\left\|\omega_{i}+x_{i}-\bar{x}\right\| \leq\left\|x_{i}\right\|+\left\|\omega_{i}-\bar{x}\right\|<$ $\alpha \delta_{1}^{\prime}+\delta_{2}^{\prime} \leq \delta_{2}$, or $\omega_{i}+x_{i} \in B_{\delta_{2}}(\bar{x})(i=1, \ldots, n)$. By (i), condition (3) is satisfied, and consequently, $\left\{\Omega_{1}, \ldots, \Omega_{n}\right\}$ is $\alpha$-transversal at $\bar{x}$ with $\delta_{1}^{\prime}$ and $\delta_{2}^{\prime}$. 
Remark 3 (i) The inequality $\alpha \delta_{1}^{\prime}+\delta_{2}^{\prime} \leq \delta_{2}$ in the last part of Proposition 5 and some statements below can be replaced by the equality $\alpha \delta_{1}^{\prime}+\delta_{2}^{\prime}=\delta_{2}$ providing in a sense the best estimate for the values of the parameters $\delta_{1}^{\prime}$ and $\delta_{2}^{\prime}$.

(ii) Each of the conditions (i)-(iii) in Proposition 5 can serve as an equivalent definition of the $\alpha$-transversality property. For the estimates derived in the subsequent statements in this paper, such equivalent definitions can have some advantages compared to the original one in Definition 1(iii). In particular, they allow to reduce some conventional proofs to a few lines.

In the convex setting, the requirements that the relations in parts (i) and (iii) of Definition 1 must hold for all small $\rho>0$ can be significantly relaxed .

Proposition 6 Let $\Omega_{1}, \ldots, \Omega_{n}$ be convex subsets of a normed space $X, \bar{x} \in \cap_{i=1}^{n} \Omega_{i}$, and $\alpha>0$. The collection $\left\{\Omega_{1}, \ldots, \Omega_{n}\right\}$ is

(i) $\alpha$-semitransversal at $\bar{x}$ with some $\delta>0$ if and only if

$$
\bigcap_{i=1}^{n}\left(\Omega_{i}-x_{i}\right) \cap B_{\delta}(\bar{x}) \neq \emptyset
$$

for all $x_{i} \in X(i=1, \ldots, n)$ with $\max _{1 \leq i \leq n}\left\|x_{i}\right\|<\alpha \delta$.

(ii) $\alpha$-transversal at $\bar{x}$ with some $\delta_{1}>0$ and $\delta_{2}>0$ if and only if

$$
\bigcap_{i=1}^{n}\left(\Omega_{i}-\omega_{i}-x_{i}\right) \cap\left(\delta_{1} \mathbb{B}\right) \neq \emptyset
$$

for all $\omega_{i} \in \Omega_{i} \cap B_{\delta_{2}}(\bar{x})$ and $x_{i} \in X(i=1, \ldots, n)$ with $\max _{1 \leq i \leq n}\left\|x_{i}\right\|<\alpha \delta_{1}$.

Proof (i) If $\left\{\Omega_{1}, \ldots, \Omega_{n}\right\}$ is $\alpha$-semitransversal at $\bar{x}$ with some $\delta>0$, then, by Definition 1(i), for all $x_{i} \in X(i=1, \ldots, n)$ with $\max _{1 \leq i \leq n}\left\|x_{i}\right\|<\alpha \delta$, and any number $\rho$ satisfying $\alpha^{-1} \max _{1 \leq i \leq n}\left\|x_{i}\right\|<\rho<\delta$, condition (1) holds. The latter condition obviously implies condition (6).

Conversely, suppose that, for some $\delta>0$, condition (6) is satisfied for all $x_{i} \in X \quad(i=1, \ldots, n)$ with $\max _{1 \leq i \leq n}\left\|x_{i}\right\|<\alpha \delta$. Let $\left.\rho \in\right] 0, \delta\left[\right.$ and $x_{i} \in X \quad(i=$ $1, \ldots, n)$ with $\max _{1 \leq i \leq n}\left\|x_{i}\right\|<\alpha \rho$. Set $t:=\rho / \delta$ and $x_{i}^{\prime}:=x_{i} / t(i=1, \ldots, n)$. Then $0<t<1$ and $\left\|x_{i}^{\prime}\right\|=\left\|x_{i}\right\| / t<\alpha \delta(i=1, \ldots, n)$, and consequently, there exists an $x^{\prime} \in \cap_{i=1}^{n}\left(\Omega_{i}-x_{i}^{\prime}\right) \cap B_{\delta}(\bar{x})$, i.e. $x^{\prime} \in B_{\delta}(\bar{x})$ and $x^{\prime}=\omega_{i}-x_{i}^{\prime}$ for some $\omega_{i} \in \Omega_{i}$, or equivalently, $x_{i}=t\left(\omega_{i}-x^{\prime}\right)(i=1, \ldots, n)$. In view of the convexity of the sets, we have $t \omega_{i}+(1-t) \bar{x} \in \Omega_{i}(i=1, \ldots, n)$. Set $x:=\bar{x}+t\left(x^{\prime}-\bar{x}\right)$. We have $x=t \omega_{i}+(1-t) \bar{x}-$ $t\left(\omega_{i}-x^{\prime}\right) \in \Omega_{i}-x_{i}(i=1, \ldots, n)$. Moreover, $\|x-\bar{x}\|=t\left\|x^{\prime}-\bar{x}\right\|<\rho$. Hence, condition (1) is satisfied. By Definition 1(i), $\left\{\Omega_{1}, \ldots, \Omega_{n}\right\}$ is $\alpha$-semitransversal at $\bar{x}$ with $\delta$.

(ii) If $\left\{\Omega_{1}, \ldots, \Omega_{n}\right\}$ is $\alpha$-transversal at $\bar{x}$ with some $\delta_{1}>0$ and $\delta_{2}>0$, then, by Definition 1(iii), for any $\omega_{i} \in \Omega_{i} \cap B_{\delta_{2}}(\bar{x})$ and $x_{i} \in X(i=1, \ldots, n)$ with $\max _{1 \leq i \leq n}\left\|x_{i}\right\|<\alpha \delta_{1}$, and any number $\rho$ satisfying $\alpha^{-1} \max _{1 \leq i \leq n}\left\|x_{i}\right\|<\rho<\delta_{1}$, condition (3) holds. The latter condition obviously implies condition (7).

Conversely, suppose that, for some $\delta_{1}>0$ and $\delta_{2}>0$, condition (7) is satisfied for all $\omega_{i} \in \Omega_{i} \cap B_{\delta_{2}}(\bar{x})$ and $x_{i} \in X(i=1, \ldots, n)$ with $\max _{1 \leq i \leq n}\left\|x_{i}\right\|<\alpha \delta_{1}$. Then the collection of convex sets $\Omega_{i}-\omega_{i}(i=1, \ldots, n)$, considered near their common point 0 , satisfies the conditions in part (i) and is consequently $\alpha$-semitransversal at 0 with $\delta_{1}$ uniformly over $\omega_{i} \in \Omega_{i} \cap B_{\delta_{2}}(\bar{x})(i=1, \ldots, n)$. This means that $\left\{\Omega_{1}, \ldots, \Omega_{n}\right\}$ is $\alpha$-transversal at $\bar{x}$ with $\delta_{1}$ and $\delta_{2}$.

Remark 4 We have not succeeded to identify a meaningful relaxation of the $\alpha$-subtransversality property in the convex setting.

Employing the same arguments as in the proof of Proposition 6, it is easy to show that in the convex case the alternative representations of $\alpha$-transversality in Proposition 5 can also be simplified. 
Proposition 7 Let $\Omega_{1}, \ldots, \Omega_{n}$ be convex subsets of a normed space $X, \bar{x} \in \cap_{i=1}^{n} \Omega_{i}, \alpha>0$, $\delta_{1}>0$ and $\delta_{2}>0$. Conditions (i)-(iii) in Proposition 5 are satisfied if and only if the following equivalent conditions hold:

(i) condition (7) is satisfied for all $\omega_{i} \in \Omega_{i}, x_{i} \in X$ with $\omega_{i}+x_{i} \in B_{\delta_{2}}(\bar{x})(i=1, \ldots, n)$ and $\max _{1 \leq i \leq n}\left\|x_{i}\right\|<\alpha \delta_{1}$;

(ii) $\cap_{i=1}^{n}\left(\Omega_{i}-x_{i}\right) \cap B_{\delta_{1}}(\bar{x}) \neq \emptyset$ for all $x_{i} \in \delta_{2} \mathbb{B}(i=1, \ldots, n)$ with $\max _{1 \leq i \leq n} d\left(\bar{x}, \Omega_{i}-x_{i}\right)<$ $\alpha \delta_{1}$

(iii) $\cap_{i=1}^{n}\left(\Omega_{i}-x_{i}\right) \cap B_{\delta_{1}}(x) \neq \emptyset$ for all $x, x_{i} \in X$ with $x+x_{i} \in B_{\delta_{2}}(\bar{x})(i=1, \ldots, n)$ and $\max _{1 \leq i \leq n} d\left(x, \Omega_{i}-x_{i}\right)<\alpha \delta_{1}$.

When the sets are convex, the semitransversality and transversality properties are equivalent.

Proposition 8 Let $\Omega_{1}, \ldots, \Omega_{n}$ be convex subsets of a normed space $X, \bar{x} \in \cap_{i=1}^{n} \Omega_{i}$, and $\alpha>0$. If $\left\{\Omega_{1}, \ldots, \Omega_{n}\right\}$ is $\alpha$-semitransversal at $\bar{x}$ with some $\delta>0$, then, for any $\left.\alpha^{\prime} \in\right] 0, \alpha$, it is $\alpha^{\prime}$-transversal at $\bar{x}$ with $\delta_{1}:=\delta$ and $\delta_{2}:=\left(\alpha-\alpha^{\prime}\right) \delta$.

As a consequence, $\left\{\Omega_{1}, \ldots, \Omega_{n}\right\}$ is semitransversal at $\bar{x}$ if and only if it is transversal at $\bar{x}$, and $\mathrm{s} \operatorname{tr}\left[\Omega_{1}, \ldots, \Omega_{n}\right](\bar{x})=\operatorname{tr}\left[\Omega_{1}, \ldots, \Omega_{n}\right](\bar{x})$.

Proof Let $\left\{\Omega_{1}, \ldots, \Omega_{n}\right\}$ be $\alpha$-semitransversal at $\bar{x}$ with some $\delta>0$, and let $\left.\alpha^{\prime} \in\right] 0, \alpha$, $\delta_{1}:=\delta$ and $\delta_{2}:=\left(\alpha-\alpha^{\prime}\right) \delta$. Let $\omega_{i} \in \Omega_{i} \cap B_{\delta_{2}}(\bar{x})$ and $x_{i} \in X(i=1, \ldots, n)$ with $\max _{1 \leq i \leq n}\left\|x_{i}\right\|<\alpha^{\prime} \delta$. Set $x_{i}^{\prime}:=\omega_{i}+x_{i}-\bar{x}(i=1, \ldots, n)$. Then $\left\|x_{i}^{\prime}\right\| \leq\left\|\omega_{i}-\bar{x}\right\|+\left\|x_{i}\right\|<$ $\delta_{2}+\alpha^{\prime} \delta=\alpha \delta(i=1, \ldots, n)$, and by Proposition 6(i), $\cap_{i=1}^{n}\left(\Omega_{i}-x_{i}^{\prime}\right) \cap B_{\delta}(\bar{x}) \neq \emptyset$, which is equivalent to condition (7). By Proposition 6(ii), $\left\{\Omega_{1}, \ldots, \Omega_{n}\right\}$ is $\alpha^{\prime}$-transversal at $\bar{x}$ with $\delta_{1}$ and $\delta_{2}$. Since $\alpha^{\prime}$ can be chosen arbitrarily close to $\alpha$, we have $\operatorname{setr}\left[\Omega_{1}, \ldots, \Omega_{n}\right](\bar{x}) \leq$ $\operatorname{tr}\left[\Omega_{1}, \ldots, \Omega_{n}\right](\bar{x})$. In view of Proposition 2 , this inequality actually holds as equality.

Remark 5 (i) Proposition 8 strengthens [19, Proposition 13(iv)].

(ii) In view of Propositions 2 and 8, in the convex case, semitransversality is in general stronger than subtransversality.

\section{Metric Characterizations}

The transversality properties of collections of sets in Definition 1 admit equivalent characterizations in metric terms.

Theorem 2 Let $\Omega_{1}, \ldots, \Omega_{n}$ be subsets of a normed space $X, \bar{x} \in \cap_{i=1}^{n} \Omega_{i}$, and $\alpha>0$. The collection $\left\{\Omega_{1}, \ldots, \Omega_{n}\right\}$ is

(i) $\alpha$-semitransversal at $\bar{x}$ with some $\delta>0$ if and only if

$$
\alpha d\left(\bar{x}, \bigcap_{i=1}^{n}\left(\Omega_{i}-x_{i}\right)\right) \leq \max _{1 \leq i \leq n}\left\|x_{i}\right\|
$$

for all $x_{i} \in X(i=1, \ldots, n)$ with $\max _{1 \leq i \leq n}\left\|x_{i}\right\|<\alpha \delta$;

(ii) $\alpha$-subtransversal at $\bar{x}$ with some $\delta_{1}>0$ and $\delta_{2}>0$ if and only if the following equivalent conditions hold:

(a) for all $x \in B_{\delta_{2}}(\bar{x})$ with $\max _{1 \leq i \leq n} d\left(x, \Omega_{i}\right)<\alpha \delta_{1}$, it holds

$$
\alpha d\left(x, \bigcap_{i=1}^{n} \Omega_{i}\right) \leq \max _{1 \leq i \leq n} d\left(x, \Omega_{i}\right) ;
$$

(b) for all $\omega_{i} \in \Omega_{i}$ and $x_{i} \in X(i=1, \ldots, n)$ with $\max _{1 \leq i \leq n}\left\|x_{i}\right\|<\alpha \delta_{1}$ and $\omega_{1}+x_{1}=$ $\ldots=\omega_{n}+x_{n} \in B_{\delta_{2}}(\bar{x})$, it holds

$$
\alpha d\left(0, \bigcap_{i=1}^{n}\left(\Omega_{i}-\omega_{i}-x_{i}\right)\right) \leq \max _{1 \leq i \leq n}\left\|x_{i}\right\| ;
$$


(iii) $\alpha$-transversal at $\bar{x}$ with some $\delta_{1}>0$ and $\delta_{2}>0$ if and only if inequality (10) is satisfied for all $\omega_{i} \in \Omega_{i} \cap B_{\delta_{2}}(\bar{x})$ and $x_{i} \in X(i=1, \ldots, n)$ with $\max _{1 \leq i \leq n}\left\|x_{i}\right\|<\alpha \delta_{1}$.

Proof (i) By Definition 1(i), $\left\{\Omega_{1}, \ldots, \Omega_{n}\right\}$ is $\alpha$-semitransversal at $\bar{x}$ with some $\delta>0$ if and only if $d\left(\bar{x}, \cap_{i=1}^{n}\left(\Omega_{i}-x_{i}\right)\right)<\rho$ for all $x_{i} \in X(i=1, \ldots, n)$ with $\max _{1 \leq i \leq n}\left\|x_{i}\right\|<\alpha \delta$ and all numbers $\rho>\alpha^{-1} \max _{1 \leq i \leq n}\left\|x_{i}\right\|$. The conclusion follows.

(ii) We first prove the equivalence between (a) and (b). Suppose condition (a) is satisfied. Let $\omega_{i} \in \Omega_{i}$ and $x_{i} \in X(i=1, \ldots, n)$ with $\max _{1 \leq i \leq n}\left\|x_{i}\right\|<\alpha \delta_{1}$ and $x:=\omega_{1}+x_{1}=$ $\ldots=\omega_{n}+x_{n} \in B_{\delta_{2}}(\bar{x})$. Then

$$
d\left(x, \Omega_{i}\right)=d\left(\omega_{i}+x_{i}, \Omega_{i}\right) \leq\left\|x_{i}\right\|<\alpha \delta_{1}(i=1, \ldots, n),
$$

and consequently, inequality (9) is satisfied. Hence,

$$
\alpha d\left(0, \bigcap_{i=1}^{n}\left(\Omega_{i}-\omega_{i}-x_{i}\right)\right)=\alpha d\left(x, \bigcap_{i=1}^{n} \Omega_{i}\right) \leq \max _{1 \leq i \leq n} d\left(x, \Omega_{i}\right) \leq \max _{1 \leq i \leq n}\left\|x_{i}\right\| .
$$

Suppose condition (b) is satisfied. Let $x \in B_{\delta_{2}}(\bar{x})$ with $\max _{1 \leq i \leq n} d\left(x, \Omega_{i}\right)<\alpha \delta_{1}$. Let $\omega_{i} \in \Omega_{i}(i=1, \ldots, n)$ with $\max _{1 \leq i \leq n}\left\|x-\omega_{i}\right\|<\alpha \delta_{1}$. Set $x_{i}^{\prime}:=x-\omega_{i}(i=1, \ldots, n)$. Then $x=x_{i}^{\prime}+\omega_{i} \in B_{\delta_{2}}(\bar{x})(i=1, \ldots, n)$ and $\max _{1 \leq i \leq n}\left\|x_{i}^{\prime}\right\|<\alpha \delta_{1}$. In view of inequality (10) with $x_{i}^{\prime}$ in place of $x_{i}(i=1, \ldots, n)$, we obtain

$$
\alpha d\left(x, \bigcap_{i=1}^{n} \Omega_{i}\right) \leq \max _{1 \leq i \leq n}\left\|x-\omega_{i}\right\| .
$$

Taking infimum in the right-hand side over $\omega_{i} \in \Omega_{i}(i=1, \ldots, n)$, we arrive at inequality (9).

We now show that $\alpha$-subtransversality is equivalent to condition (a). By Definition 1(ii), $\left\{\Omega_{1}, \ldots, \Omega_{n}\right\}$ is $\alpha$-subtransversal at $\bar{x}$ with some $\delta_{1}>0$ and $\delta_{2}>0$ if and only if $d\left(x, \cap_{i=1}^{n} \Omega_{i}\right)<\rho$ for all $x \in B_{\delta_{2}}(\bar{x})$ with $\max _{1 \leq i \leq n} d\left(x, \Omega_{i}\right)<\alpha \delta_{1}$ and all numbers $\rho>\alpha^{-1} \max _{1 \leq i \leq n} d\left(x, \Omega_{i}\right)$. The conclusion follows.

(iii) By the Definition 1(iii), $\left\{\Omega_{1}, \ldots, \Omega_{n}\right\}$ is $\alpha$-transversal at $\bar{x}$ with some $\delta_{1}>0$ and $\delta_{2}>0$ if and only if $d\left(0, \cap_{i=1}^{n}\left(\Omega_{i}-\omega_{i}-x_{i}\right)\right)<\rho$ for all $\omega_{i} \in \Omega_{i} \cap B_{\delta_{2}}(\bar{x})$ and $x_{i} \in X$ $(i=1, \ldots, n)$ with $\max _{1 \leq i \leq n}\left\|x_{i}\right\|<\alpha \delta_{1}$, and all numbers $\rho>\alpha^{-1} \max _{1 \leq i \leq n}\left\|x_{i}\right\|$. The conclusion follows.

The alternative metric characterizations of $\alpha$-transversality in the next statement correspond to the respective geometric properties in Proposition 5.

Proposition 9 Let $\Omega_{1}, \ldots, \Omega_{n}$ be subsets of a normed space $X, \bar{x} \in \cap_{i=1}^{n} \Omega_{i}, \alpha>0, \delta_{1}>0$ and $\delta_{2}>0$. The following conditions are equivalent:

(i) inequality (10) holds for all $\omega_{i} \in \Omega_{i}, x_{i} \in X$ with $\omega_{i}+x_{i} \in B_{\delta_{2}}(\bar{x})(i=1, \ldots, n)$ and $\max _{1 \leq i \leq n}\left\|x_{i}\right\|<\alpha \delta_{1}$;

(ii) for all $x_{i} \in \delta_{2} \mathbb{B}(i=1, \ldots, n)$ with $\max _{1 \leq i \leq n} d\left(\bar{x}, \Omega_{i}-x_{i}\right)<\alpha \delta_{1}$, it holds

$$
\alpha d\left(\bar{x}, \bigcap_{i=1}^{n}\left(\Omega_{i}-x_{i}\right)\right) \leq \max _{1 \leq i \leq n} d\left(\bar{x}, \Omega_{i}-x_{i}\right) ;
$$

(iii) for all $x, x_{i} \in X$ with $x+x_{i} \in B_{\delta_{2}}(\bar{x})(i=1, \ldots, n)$ and $\max _{1 \leq i \leq n} d\left(x, \Omega_{i}-x_{i}\right)<\alpha \delta_{1}$, it holds

$$
\alpha d\left(x, \bigcap_{i=1}^{n}\left(\Omega_{i}-x_{i}\right)\right) \leq \max _{1 \leq i \leq n} d\left(x, \Omega_{i}-x_{i}\right) .
$$

Moreover, if $\left\{\Omega_{1}, \ldots, \Omega_{n}\right\}$ is $\alpha$-transversal at $\bar{x}$ with some $\delta_{1}>0$ and $\delta_{2}>0$, then conditions (i)-(iii) hold with any $\left.\left.\delta_{1}^{\prime} \in\right] 0, \delta_{1}\right]$ and $\delta_{2}^{\prime}>0$ satisfying $\alpha \delta_{1}^{\prime}+\delta_{2}^{\prime} \leq \delta_{2}$ in place of $\delta_{1}$ and $\delta_{2}$.

Conversely, if conditions (i)-(iii) hold with some $\delta_{1}>0$ and $\delta_{2}>0$, then $\left\{\Omega_{1}, \ldots, \Omega_{n}\right\}$ is $\alpha$-transversal at $\bar{x}$ with any $\left.\left.\delta_{1}^{\prime} \in\right] 0, \delta_{1}\right]$ and $\delta_{2}^{\prime}>0$ satisfying $\alpha \delta_{1}^{\prime}+\delta_{2}^{\prime} \leq \delta_{2}$. 
Proof The statement is a consequence of Proposition 5. It suffices to notice that inequality (10) is equivalent to condition (3) satisfied for any $\rho>\alpha^{-1} \max _{1 \leq i \leq n}\left\|x_{i}\right\|$; inequality (11) is equivalent to condition (1) satisfied for any $\rho>\alpha^{-1} \max _{1 \leq i \leq n} d\left(\bar{x}, \Omega_{i}-x_{i}\right)$, and inequality (12) is equivalent to condition (5) satisfied for any $\rho>\alpha^{-1} \max _{1 \leq i \leq n} d\left(x, \Omega_{i}-x_{i}\right)$.

Remark 6 Condition (12) served as the main metric characterization of transversality in $[19,20]$ and subsequent publications. Condition (11) has been picked up recently in [7]. This condition seems an important advancement as it replaces an arbitrary point $x$ with the given reference point $\bar{x}$. Condition (10) seems new. In accordance with Theorem 2(iii), it is the most straightforward metric counterpart of the original geometric property (3).

In the convex case, the estimates in parts (i) and (iii) of Theorem 2 can be simplified. The next statement is a direct consequence of Proposition 6.

Proposition 10 Let $\Omega_{1}, \ldots, \Omega_{n}$ be convex subsets of a normed vector space $X, \bar{x} \in \cap_{i=1}^{n} \Omega_{i}$, and $\alpha>0$. The collection $\left\{\Omega_{1}, \ldots, \Omega_{n}\right\}$ is

(i) $\alpha$-semitransversal at $\bar{x}$ with some $\delta>0$ if and only if $d\left(\bar{x}, \cap_{i=1}^{n}\left(\Omega_{i}-x_{i}\right)\right)<\delta$ for all $x_{i} \in X(i=1, \ldots, n)$ with $\max _{1 \leq i \leq n}\left\|x_{i}\right\|<\alpha \delta$

(ii) $\alpha$-transversal at $\bar{x}$ with some $\delta_{1}>0$ and $\delta_{2}>0$ if and only if $d\left(0, \cap_{i=1}^{n}\left(\Omega_{i}-\omega_{i}-x_{i}\right)\right)<\delta_{1}$ for all $\omega_{i} \in \Omega_{i} \cap B_{\delta_{2}}(\bar{x})$ and $x_{i} \in X \quad(i=1, \ldots, n)$ with $\max _{1 \leq i \leq n}\left\|x_{i}\right\|<\alpha \delta_{1}$.

Remark 7 The alternative metric characterizations of $\alpha$-transversality in Proposition 9 admit similar simplifications.

\section{Transversality and Regularity}

In this section, we clarify quantitative relations between transversality properties of collections of sets and the corresponding regularity properties of set-valued mappings.

The next definition is a modification of [29, Definition 5.1].

Definition 2 Let $X$ and $Y$ be metric spaces, $F: X \rightrightarrows Y,(\bar{x}, \bar{y}) \in \operatorname{gph} F$, and $\alpha>0$. The mapping $F$ is

(i) $\alpha$-semiregular at $(\bar{x}, \bar{y})$ if there exists a $\delta>0$ such that

$$
\alpha d\left(\bar{x}, F^{-1}(y)\right) \leq d(y, \bar{y})
$$

for all $y \in B_{\alpha \delta}(\bar{y})$

(ii) $\alpha$-subregular at $(\bar{x}, \bar{y})$ if there exist $\delta_{1}>0$ and $\delta_{2}>0$ such that

$$
\alpha d\left(x, F^{-1}(\bar{y})\right) \leq d(\bar{y}, F(x))
$$

for all $x \in B_{\delta_{2}}(\bar{x})$ with $d(\bar{y}, F(x))<\alpha \delta_{1}$;

(iii) $\alpha$-regular at $(\bar{x}, \bar{y})$ if there exist $\delta_{1}>0$ and $\delta_{2}>0$ such that

$$
\alpha d\left(x, F^{-1}(y)\right) \leq d(y, F(x))
$$

for all $x \in X$ and $y \in Y$ with $d(x, \bar{x})+d(y, \bar{y})<\delta_{2}$ and $d(y, F(x))<\alpha \delta_{1}$.

The number $\alpha>0$ in each of the properties in Definition 2 plays the role of a rate of the respective property. The exact upper bound of all $\alpha>0$ such that the property holds with some $\delta>0$, or $\delta_{1}>0$ and $\delta_{2}>0$, is called the modulus of this property. We use notations $\mathrm{s}_{\mathrm{e}} \operatorname{rg}[F](\bar{x}, \bar{y}), \operatorname{srg}[F](\bar{x}, \bar{y})$ and $\operatorname{rg}[F](\bar{x}, \bar{y})$ for the moduli of the respective properties. If a property does not hold, then by convention the respective modulus equals 0 .

If $F$ is $\alpha$-semiregular (respectively, $\alpha$-subregular or $\alpha$-regular) at $(\bar{x}, \bar{y})$ with some $\alpha>0$ and $\delta>0$ (respectively, $\delta_{1}>0$ and $\delta_{2}>0$ ), we often simply say that $F$ is semiregular (respectively, subregular or regular) at $(\bar{x}, \bar{y})$. 
The last two regularity properties in Definition 2 have been very well studied for decades due to their numerous important applications; see, e.g., monographs $[14,17,18$, 33]. Note the notations $\operatorname{subreg}(F ; \bar{x} \mid \bar{y})$ and $\operatorname{reg}(F ; \bar{x} \mid \bar{y})$ often used for the respective moduli (cf. [14]), as well as an important difference in their definitions which is reflected in the relations:

$$
\operatorname{srg}[F](\bar{x}, \bar{y})=\frac{1}{\operatorname{subreg}(F ; \bar{x} \mid \bar{y})}, \quad \operatorname{rg}[F](\bar{x}, \bar{y})=\frac{1}{\operatorname{reg}(F ; \bar{x} \mid \bar{y})} .
$$

In fact, $\operatorname{rg}[F](\bar{x}, \bar{y})$ coincides with the modulus of surjection [17]. Unlike its more famous siblings, the first property in Definition 2 has only recently started attracting attention of researchers; see $[1,8,21]$.

As in the case of Definition 1 of the transversality properties, the role of the $\delta$ 's in the above definitions is more technical: they control the size of the neighbourhoods involved in the respective definitions. Of course, if a property in part (ii) or (iii) is satisfied with some $\delta_{1}>0$ and $\delta_{2}>0$, it is also satisfied with the single $\delta:=\min \left\{\delta_{1}, \delta_{2}\right\}$ in place of both $\delta_{1}$ and $\delta_{2}$. Moreover, in this case (or more generally, when $\delta_{1} \geq \delta_{2}$ ) the inequality $d(\bar{y}, F(x))<\alpha \delta_{1}$ in part (ii) and the inequality $d(y, F(x))<\alpha \delta_{1}$ in part (iii) can be dropped. We use here two different parameters to emphasise their different roles in the definitions and the corresponding characterizations, and expose connections with the transversality properties in Definition 1.

It is immediate from the definition that, if a mapping is $\alpha$-regular at a point in its graph, it is also $\alpha$-semiregular and $\alpha$-subregular at this point; hence, $\operatorname{se} \operatorname{rg}[F](\bar{x}, \bar{y}) \geq$ $\operatorname{rg}[F](\bar{x}, \bar{y})$ and $\operatorname{srg}[F](\bar{x}, \bar{y}) \geq \operatorname{rg}[F](\bar{x}, \bar{y})$ for any $(\bar{x}, \bar{y}) \in \operatorname{gph} F$.

Note the combined inequality $d(x, \bar{x})+d(y, \bar{y})<\delta_{2}$ employed in (13) instead of the more traditional separate conditions $x \in B_{\delta_{2}}(\bar{x})$ and $y \in B_{\delta_{2}}(\bar{y})$. This replacement does not affect the property of metric $\alpha$-regularity itself, but can have an effect on the value of $\delta_{2}$ which ensures the property. Employing this inequality in (13) is convenient for establishing relations between the regularity and transversality properties.

Now we return to our main setting of a collection of $n \geq 2$ subsets $\Omega_{1}, \ldots, \Omega_{n}$ of a normed space $X$, having a common point $\bar{x} \in \cap_{i=1}^{n} \Omega_{i}$, and consider a set-valued mapping $F: X \rightrightarrows X^{n}$ given by

$$
F(x):=\left(\Omega_{1}-x\right) \times \ldots \times\left(\Omega_{n}-x\right), \quad x \in X,
$$

which is going to play the key role in establishing relations between the regularity and transversality properties. It was most likely first used by Ioffe in [16]. Observe that $\bar{y}:=(0, \ldots, 0) \in F(\bar{x})$ and

$$
F^{-1}\left(x_{1}, \ldots, x_{n}\right)=\left(\Omega_{1}-x_{1}\right) \cap \ldots \cap\left(\Omega_{n}-x_{n}\right), \quad x_{1}, \ldots, x_{n} \in X .
$$

Recall that the space $Y:=X^{n}$ is equipped with the maximum norm.

The next statement is a reformulation of Theorem 2 .

Theorem 3 Let $\Omega_{1}, \ldots, \Omega_{n}$ be subsets of a normed space $X, \bar{x} \in \cap_{i=1}^{n} \Omega_{i}, \alpha>0$, $F: X \rightrightarrows X^{n}$ be defined by (14), and $\bar{y}:=(0, \ldots, 0) \in X^{n}$. The collection $\left\{\Omega_{1}, \ldots, \Omega_{n}\right\}$ is

(i) $\alpha$-semitransversal at $\bar{x}$ with some $\delta>0$ if and only if $F$ is $\alpha$-semiregular at $(\bar{x}, \bar{y})$ with $\delta$;

(ii) $\alpha$-subtransversal at $\bar{x}$ with some $\delta_{1}>0$ and $\delta_{2}>0$ if and only if $F$ is $\alpha$-subregular at $(\bar{x}, \bar{y})$ with $\delta_{1}$ and $\delta_{2}$;

(iii) $\alpha$-transversal at $\bar{x}$ with some $\delta_{1}>0$ and $\delta_{2}>0$ if and only if

$$
\alpha d\left(0, F^{-1}\left(\omega_{1}+x_{1}, \ldots, \omega_{n}+x_{n}\right)\right) \leq\|y\|
$$

for all $\omega_{i} \in \Omega_{i} \cap B_{\delta_{2}}(\bar{x})(i=1, \ldots, n)$ and $y:=\left(x_{1}, \ldots, x_{n}\right) \in X^{n}$ with $\|y\|<\alpha \delta_{1}$.

Thanks to parts (i) and (ii) of Theorem 3, we have the exact equivalences between the $\alpha$-semitransversality and $\alpha$-subtransversality of the collection $\left\{\Omega_{1}, \ldots, \Omega_{n}\right\}$ on one hand, and the respective $\alpha$-semiregularity and $\alpha$-subregularity of the mapping $F$ on the other hand. However, we do not seem to have the exact equivalence between the remaining 
two properties, at least quantitatively, as condition (15) is not exactly of the form (13). Fortunately, Proposition 9 resolves the issue. The next proposition shows that the conventional $\alpha$-regularity property in Definition 2(iii) is not a direct counterpart of the conventional $\alpha$-transversality property in Definition 1(iii), but rather of its alternative representation in Proposition 9(iii).

Proposition 11 Let $\Omega_{1}, \ldots, \Omega_{n}$ be subsets of a normed space $X, \bar{x} \in \cap_{i=1}^{n} \Omega_{i}, \alpha>0$, $F: X \rightrightarrows X^{n}$ be defined by (14), $\bar{y}:=(0, \ldots, 0) \in X^{n}, \delta_{1}>0$ and $\delta_{2}>0$. The following conditions are equivalent:

(i) inequality (15) holds for all $\omega_{i} \in \Omega_{i}(i=1, \ldots, n), y:=\left(x_{1}, \ldots, x_{n}\right) \in X^{n}$ with $\omega_{i}+x_{i} \in B_{\delta_{2}}(\bar{x})(i=1, \ldots, n)$ and $\|y\|<\alpha \delta_{1}$;

(ii) for all $y \in \delta_{2} \mathbb{B}_{X^{n}}$ with $d(y, F(\bar{x}))<\alpha \delta_{1}$, it holds

$$
\alpha d\left(\bar{x}, F^{-1}(y)\right) \leq d(y, F(\bar{x}))
$$

(iii) inequality (13) holds for all $x \in X, y:=\left(x_{1}, \ldots, x_{n}\right) \in X^{n}$ with $x+x_{i} \in B_{\delta_{2}}(\bar{x})$ $(i=1, \ldots, n)$ and $d(y, F(x))<\alpha \delta_{1}$;

(iv) the mapping $F$ is $\alpha$-regular at $(\bar{x}, \bar{y})$ with $\delta_{1}$ and $\delta_{2}$.

Moreover, if $\left\{\Omega_{1}, \ldots, \Omega_{n}\right\}$ is $\alpha$-transversal at $\bar{x}$ with some $\delta_{1}>0$ and $\delta_{2}>0$, then conditions (i)-(iv) hold with any $\left.\left.\delta_{1}^{\prime} \in\right] 0, \delta_{1}\right]$ and $\delta_{2}^{\prime}>0$ satisfying $\alpha \delta_{1}^{\prime}+\delta_{2}^{\prime} \leq \delta_{2}$ in place of $\delta_{1}$ and $\delta_{2}$.

Conversely, if conditions (i)-(iv) hold with some $\delta_{1}>0$ and $\delta_{2}>0$, then $\left\{\Omega_{1}, \ldots, \Omega_{n}\right\}$ is $\alpha$-transversal at $\bar{x}$ with any $\left.\left.\delta_{1}^{\prime} \in\right] 0, \delta_{1}\right]$ and $\delta_{2}^{\prime}>0$ satisfying $\alpha \delta_{1}^{\prime}+\delta_{2}^{\prime} \leq \delta_{2}$.

Proof With the exception of item (iv), the statement is a reformulation of Proposition 9 in terms of the mapping $F$. It is easy to see that conditions (iii) and (iv) are equivalent.

(iii) $\Rightarrow$ (iv). It suffices to notice that condition $\|x-\bar{x}\|+\|y-\bar{y}\|<\delta_{2}$ with $x \in X$ and $y:=\left(x_{1}, \ldots, x_{n}\right) \in X^{n}$ implies $x+x_{i} \in B_{\delta_{2}}(\bar{x})(i=1, \ldots, n)$.

(iv) $\Rightarrow$ (iii). If $x+x_{i} \in B_{\delta_{2}}(\bar{x})(i=1, \ldots, n)$ for some $x \in X$ and $y:=\left(x_{1}, \ldots, x_{n}\right) \in X^{n}$, we can set $x_{i}^{\prime}:=x+x_{i}-\bar{x}(i=1, \ldots, n)$ and $y^{\prime}:=\left(x_{1}^{\prime}, \ldots, x_{n}^{\prime}\right)$. Then obviously $d\left(y^{\prime}, F(\bar{x})\right)=$ $d(y, F(x)), d\left(\bar{x}, F^{-1}\left(y^{\prime}\right)\right)=d\left(x, F^{-1}(y)\right)$, and $\left\|y^{\prime}-\bar{y}\right\|<\delta_{2}$. Condition (iii) is a consequence of the one in Definition 2(iii) with $\bar{x}$ and $y^{\prime}$ in place of $x$ and $y$, respectively.

Remark 8 Thanks to Proposition 11, we have equivalence between the $\alpha$-transversality property of $\left\{\Omega_{1}, \ldots, \Omega_{n}\right\}$ and the $\alpha$-regularity property of $F$, although not necessarily with the same $\delta_{1}$ and $\delta_{2}$. The three conditions in Proposition 11 together with condition (iii) in Theorem 3 provide a series of metric characterizations of both equivalent properties in terms of the set-valued mapping $F$. Observe also that, thanks to Proposition 11, the set-valued mapping $F$ given by (14) provides an important case when the point $x$ in the inequality (13) defining metric $\alpha$-regularity can be replaced by the fixed reference point $\bar{x}$.

The next corollary of Theorem 3 and Proposition 11 collects ' $\delta$-free' versions of the discussed equivalences. It recaptures [29, Proposition 5.1].

Corollary 1 Let $\Omega_{1}, \ldots, \Omega_{n}$ be subsets of a normed space $X, \bar{x} \in \cap_{i=1}^{n} \Omega_{i}, \alpha>0, F: X \rightrightarrows$ $X^{n}$ be defined by (14), and $\bar{y}:=(0, \ldots, 0) \in X^{n}$.

(i) $\left\{\Omega_{1}, \ldots, \Omega_{n}\right\} \alpha$-semitransversal at $\bar{x}$ if and only if $F$ is $\alpha$-semiregular at $(\bar{x}, \bar{y})$. Moreover, $\operatorname{se} \operatorname{tr}\left[\Omega_{1}, \ldots, \Omega_{n}\right](\bar{x})=\operatorname{se} \operatorname{rg}[F](\bar{x}, \bar{y})$.

(ii) $\left\{\Omega_{1}, \ldots, \Omega_{n}\right\}$ is $\alpha$-subtransversal at $\bar{x}$ if and only if $F$ is $\alpha$-subregular at $(\bar{x}, \bar{y})$. Moreover, $\operatorname{str}\left[\Omega_{1}, \ldots, \Omega_{n}\right](\bar{x})=\operatorname{srg}[F](\bar{x}, \bar{y})$.

(iii) $\left\{\Omega_{1}, \ldots, \Omega_{n}\right\}$ is $\alpha$-transversal at $\bar{x}$ if and only if $F$ is $\alpha$-regular at $(\bar{x}, \bar{y})$. Moreover, $\operatorname{tr}\left[\Omega_{1}, \ldots, \Omega_{n}\right](\bar{x})=\operatorname{rg}[F](\bar{x}, \bar{y})$.

Remark 9 In view of Proposition 8, it follows from the above corollary that, when the sets $\Omega_{1}, \ldots, \Omega_{n}$ are convex, the semiregularity and regularity properties of the set-valued mapping $F$ defined by (14) are equivalent, and $\mathrm{s}_{\mathrm{e}} \operatorname{rg}[F](\bar{x}, \bar{y})=\operatorname{rg}[F](\bar{x}, \bar{y})$. 
In view of Theorem 3 and Proposition 11, the $\alpha$-transversality properties of collections of sets can be viewed as particular cases of the corresponding $\alpha$-regularity properties of set-valued mappings. Now we are going to show that the two popular models are in a sense equivalent.

Given an arbitrary set-valued mapping $F: X \rightrightarrows Y$ between metric spaces and a point $(\bar{x}, \bar{y}) \in \operatorname{gph} F$, we can construct the two sets:

$$
\Omega_{1}:=\operatorname{gph} F, \quad \Omega_{2}:=X \times\{\bar{y}\}
$$

in the product space $X \times Y$. Note that $(\bar{x}, \bar{y}) \in \Omega_{1} \cap \Omega_{2}$. To establish relations between the regularity properties of $F$ and the transversality properties of the collection of two sets (16), we have to assume that $X$ and $Y$ are normed vector spaces.

The next theorem translates the metric characterizations of the transversality properties of collection of sets in Theorem 1 into certain metric properties of the set-valued mapping $F$.

Theorem 4 Let $X$ and $Y$ be normed spaces, $F: X \rightrightarrows Y,(\bar{x}, \bar{y}) \in \operatorname{gph} F, \Omega_{1}$ and $\Omega_{2}$ be defined by (16), and $\alpha>0$.

(i) If $\left\{\Omega_{1}, \Omega_{2}\right\}$ is $\alpha$-semitransversal at $(\bar{x}, \bar{y})$ with some $\delta>0$, then

$$
\alpha d\left(\bar{x}+u, F^{-1}(\bar{y}+v)\right) \leq \max \left\{\|u\|, \frac{1}{2}\|v\|\right\}
$$

for all $u \in(\alpha \delta) \mathbb{B}_{X}$ and $v \in(2 \alpha \delta) \mathbb{B}_{Y}$.

(ii) If $\left\{\Omega_{1}, \Omega_{2}\right\}$ is $\alpha$-subtransversal at $(\bar{x}, \bar{y})$ with some $\delta_{1}>0$ and $\delta_{2}>0$, then

$$
\alpha d\left(x, F^{-1}(\bar{y})\right) \leq \max \{d((x, y), \operatorname{gph} F),\|y-\bar{y}\|\}
$$

for all $x \in B_{\delta_{2}}(\bar{x})$ and $y \in B_{\min \left\{\alpha \delta_{1}, \delta_{2}\right\}}(\bar{y})$ with $d((x, y), \operatorname{gph} F)<\alpha \delta_{1}$.

(iii) If $\left\{\Omega_{1}, \Omega_{2}\right\}$ is $\alpha$-transversal at $(\bar{x}, \bar{y})$ with some $\delta_{1}>0$ and $\delta_{2}>0$, then

$$
\alpha d\left(x+u, F^{-1}(y+v)\right) \leq \max \left\{\|u\|, \frac{1}{2}\|v\|\right\}
$$

for all $(x, y) \in \operatorname{gph} F \cap B_{\delta_{2}}(\bar{x}, \bar{y}), u \in\left(\alpha \delta_{1}\right) \mathbb{B}_{X}$ and $v \in\left(2 \alpha \delta_{1}\right) \mathbb{B}_{Y}$.

Moreover, if $\alpha \leq 1$, then the implications in (i)-(iii) hold as equivalences.

Proof (i) Given $x_{1}:=\left(u_{1}, v_{1}\right)$ and $x_{2}:=\left(u_{2}, v_{2}\right) \in X \times Y$, we have

$$
\begin{gathered}
\left(\Omega_{1}-x_{1}\right) \cap\left(\Omega_{2}-x_{2}\right)=\left(F^{-1}\left(\bar{y}+v_{1}-v_{2}\right)-u_{1}\right) \times\left\{\bar{y}-v_{2}\right\}, \\
d\left((\bar{x}, \bar{y}),\left(\Omega_{1}-x_{1}\right) \cap\left(\Omega_{2}-x_{2}\right)\right)=\max \left\{d\left(\bar{x}+u_{1}, F^{-1}\left(\bar{y}+v_{1}-v_{2}\right)\right),\left\|v_{2}\right\|\right\} .
\end{gathered}
$$

Thus, inequality

$$
\alpha d\left((\bar{x}, \bar{y}),\left(\Omega_{1}-x_{1}\right) \cap\left(\Omega_{2}-x_{2}\right)\right) \leq \max \left\{\left\|x_{1}\right\|,\left\|x_{2}\right\|\right\}
$$

implies

$$
\alpha d\left(\bar{x}+u_{1}, F^{-1}\left(\bar{y}+v_{1}-v_{2}\right)\right) \leq \max \left\{\left\|u_{1}\right\|,\left\|u_{2}\right\|,\left\|v_{1}\right\|,\left\|v_{2}\right\|\right\},
$$

and the converse implication is true if $\alpha \leq 1$.

We claim that the following conditions are equivalent:

(a) inequality (17) holds for all $u \in(\alpha \delta) \mathbb{B}_{X}$ and $v \in(2 \alpha \delta) \mathbb{B}_{Y}$;

(b) inequality (22) holds for all $u_{1}, u_{2} \in(\alpha \delta) \mathbb{B}_{X}$ and $v_{1}, v_{2} \in(\alpha \delta) \mathbb{B}_{Y}$.

(a) $\Rightarrow$ (b). Given $u_{1}, u_{2} \in(\alpha \delta) \mathbb{B}_{X}$ and $v_{1}, v_{2} \in(\alpha \delta) \mathbb{B}_{Y}$, inequality (17) is satisfied with $u_{1}$ and $v_{1}-v_{2}$ in place of $u$ and $v$, i.e.

$$
\alpha d\left(\bar{x}+u_{1}, F^{-1}\left(\bar{y}+v_{1}-v_{2}\right)\right) \leq \max \left\{\left\|u_{1}\right\|, \frac{1}{2}\left\|v_{1}-v_{2}\right\|\right\},
$$

and consequently, inequality (22) is satisfied.

(b) $\Rightarrow$ (a). Given $u \in(\alpha \delta) \mathbb{B}_{X}$ and $v \in(2 \alpha \delta) \mathbb{B}_{Y}$, inequality (22) is satisfied with $u_{1}:=u, u_{2}:=0, v_{1}:=v / 2$ and $v_{2}:=-v / 2$, which is equivalent to inequality (17).

Hence, $(a) \Leftrightarrow(b)$, which, in view of Theorem 2(i), proves the assertion. 
(ii) Given an $(x, y) \in X \times Y$, thanks to (16), we have

$$
d\left((x, y), \Omega_{2}\right)=\|y-\bar{y}\|, d\left((x, y), \Omega_{1} \cap \Omega_{2}\right)=\max \left\{d\left(x, F^{-1}(\bar{y})\right),\|y-\bar{y}\|\right\} .
$$

Thus, inequality

$$
\alpha d\left((x, y), \Omega_{1} \cap \Omega_{2}\right) \leq \max \left\{d\left((x, y), \Omega_{1}\right), d\left((x, y), \Omega_{2}\right)\right\}
$$

implies inequality (18), and the converse implication is true if $\alpha \leq 1$. In view of Theorem 2(ii), this proves the assertion.

(iii) Given $x_{1}:=\left(u_{1}, v_{1}\right), x_{2}:=\left(u_{2}, v_{2}\right),(x, y) \in X \times Y$ and $z \in X$, we have

$$
\begin{aligned}
\left(\Omega_{1}-(x, y)-x_{1}\right) \cap\left(\Omega_{2}-\right. & \left.(z, \bar{y})-x_{2}\right) \\
& =\left(F^{-1}\left(y+v_{1}-v_{2}\right)-x-u_{1}\right) \times\left\{-v_{2}\right\}, \\
d\left((0,0),\left(\Omega_{1}-(x, y)-x_{1}\right)\right. & \left.\cap\left(\Omega_{2}-(z, \bar{y})-x_{2}\right)\right) \\
= & \max \left\{d\left(x+u_{1}, F^{-1}\left(y+v_{1}-v_{2}\right)\right),\left\|v_{2}\right\|\right\} .
\end{aligned}
$$

Thus, inequality

$$
\alpha d\left((0,0),\left(\Omega_{1}-(x, y)-x_{1}\right) \cap\left(\Omega_{2}-(z, \bar{y})-x_{2}\right)\right) \leq \max \left\{\left\|x_{1}\right\|,\left\|x_{2}\right\|\right\}
$$

implies

$$
\alpha d\left(x+u_{1}, F^{-1}\left(y+v_{1}-v_{2}\right)\right) \leq \max \left\{\left\|u_{1}\right\|,\left\|u_{2}\right\|,\left\|v_{1}\right\|,\left\|v_{2}\right\|\right\},
$$

and the converse implication is true if $\alpha \leq 1$. The same arguments as in the proof of (i) show that the last inequality holds for all $(x, y) \in \Omega_{1} \cap B_{\delta_{2}}(\bar{x}, \bar{y}), u_{1}, u_{2} \in\left(\alpha \delta_{1}\right) \mathbb{B}_{X}$ and $v_{1}, v_{2} \in\left(\alpha \delta_{1}\right) \mathbb{B}_{Y}$ if and only if inequality (19) holds for all $(x, y) \in \operatorname{gph} F \cap B_{\delta_{2}}(\bar{x}, \bar{y})$, $u \in\left(\alpha \delta_{1}\right) \mathbb{B}_{X}$ and $v \in\left(2 \alpha \delta_{1}\right) \mathbb{B}_{Y}$. In view of Theorem 2(iii), this proves the assertion.

Remark 10 If $\operatorname{gph} F$ is closed and $(\bar{x}, \bar{y}) \in \operatorname{bdgph} F$, then, in view of Proposition 4, we automatically have $\alpha \leq 1$ in parts (ii) and (iii) of Theorem 4 .

Employing the estimates established in the proof of Theorem 4, we can also translate the metric characterizations of $\alpha$-transversality in Proposition 9 into corresponding properties of the set-valued mapping $F$.

Proposition 12 Let $X$ and $Y$ be normed spaces, $F: X \rightrightarrows Y,(\bar{x}, \bar{y}) \in \operatorname{gph} F, \Omega_{1}$ and $\Omega_{2}$ be defined by (16), $\alpha>0, \delta_{1}>0$ and $\delta_{2}>0$. The following conditions are equivalent:

(i) for all $(x, y) \in \operatorname{gph} F,\left(u, v_{1}\right) \in\left(\alpha \delta_{1}\right) \mathbb{B}, v_{2} \in \min \left\{\alpha \delta_{1}, \delta_{2}\right\} \mathbb{B}$ with $(x, y)+\left(u, v_{1}\right) \in$ $B_{\delta_{2}}(\bar{x}, \bar{y})$, it holds

$$
\alpha d\left(x+u, F^{-1}\left(y+v_{1}-v_{2}\right)\right) \leq \max \left\{\|u\|,\left\|v_{1}\right\|,\left\|v_{2}\right\|\right\} ;
$$

(ii) for all $\left(u, v_{1}\right) \in \delta_{2} \mathbb{B}$ and $v_{2} \in \min \left\{\alpha \delta_{1}, \delta_{2}\right\} \mathbb{B}$ with $d\left((\bar{x}, \bar{y})+\left(u, v_{1}\right)\right.$, gph $\left.F\right)<\alpha \delta_{1}$, it holds

$$
\alpha d\left(\bar{x}+u, F^{-1}\left(\bar{y}+v_{1}-v_{2}\right)\right) \leq \max \left\{d\left((\bar{x}, \bar{y})+\left(u, v_{1}\right), \operatorname{gph} F\right),\left\|v_{2}\right\|\right\} ;
$$

(iii) for all $(x, y), \quad\left(u, v_{1}\right) \in X \times Y$ and $v_{2} \in Y$ with $(x, y)+\left(u, v_{1}\right) \in B_{\delta_{2}}(\bar{x}, \bar{y})$, $d\left((x, y)+\left(u, v_{1}\right), \operatorname{gph} F\right)<\alpha \delta_{1}$ and $\left\|y+v_{2}-\bar{y}\right\|<\min \left\{\alpha \delta_{1}, \delta_{2}\right\}$, it holds

$$
\alpha d\left(x+u, F^{-1}\left(y+v_{1}-v_{2}\right)\right) \leq \max \left\{d\left((x, y)+\left(u, v_{1}\right), \operatorname{gph} F\right),\left\|y+v_{2}-\bar{y}\right\|\right\} .
$$

Moreover, if $\left\{\Omega_{1}, \Omega_{2}\right\}$ is $\alpha$-transversal at $(\bar{x}, \bar{y})$ with some $\delta_{1}>0$ and $\delta_{2}>0$, then conditions (i)-(iii) hold with any $\left.\left.\delta_{1}^{\prime} \in\right] 0, \delta_{1}\right]$ and $\delta_{2}^{\prime}>0$ satisfying $\alpha \delta_{1}^{\prime}+\delta_{2}^{\prime} \leq \delta_{2}$ in place of $\delta_{1}$ and $\delta_{2}$.

Conversely, if $\alpha \leq 1$ and conditions (i)-(iii) hold with some $\delta_{1}>0$ and $\delta_{2}>0$, then $\left\{\Omega_{1}, \Omega_{2}\right\}$ is $\alpha$-transversal at $(\bar{x}, \bar{y})$ with any $\left.\left.\delta_{1}^{\prime} \in\right] 0, \delta_{1}\right]$ and $\delta_{2}^{\prime}>0$ satisfying $\alpha \delta_{1}^{\prime}+\delta_{2}^{\prime} \leq \delta_{2}$. 
Proof (i) Given $x_{1}:=\left(u_{1}, v_{1}\right), x_{2}:=\left(u_{2}, v_{2}\right),(x, y) \in X \times Y$ and $z \in X$, inequality (23) implies (24), and the conditions are equivalent if $\alpha \leq 1$. Moreover, given $x, u \in X$ and $y, v_{1}, v_{2} \in Y$, inequality (24) holds with $u_{1}:=u$ for all $u_{2} \in X$ with $\left\|u_{2}\right\|<\alpha \delta_{1}$ if and only if inequality (25) is satisfied. Hence, condition (i) is equivalent to Proposition 9(i).

(ii) Given $x_{1}:=\left(u_{1}, v_{1}\right)$ and $x_{2}:=\left(u_{2}, v_{2}\right)$, inequality

$$
\alpha d\left((\bar{x}, \bar{y}),\left(\Omega_{1}-x_{1}\right) \cap\left(\Omega_{2}-x_{2}\right)\right) \leq \max \left\{d\left((\bar{x}, \bar{y}), \Omega_{1}-x_{1}\right), d\left((\bar{x}, \bar{y}), \Omega_{2}-x_{2}\right)\right\}
$$

implies

$$
\alpha d\left(\bar{x}+u_{1}, F^{-1}\left(\bar{y}+v_{1}-v_{2}\right)\right) \leq \max \left\{d\left((\bar{x}, \bar{y})+\left(u_{1}, v_{1}\right), \operatorname{gph} F\right),\left\|v_{2}\right\|\right\},
$$

and the converse implication is true if $\alpha \leq 1$. Moreover, given $u \in X$ and $v_{1}, v_{2} \in Y$, inequality (28) holds with $u_{1}:=u$ if and only if inequality (26) is satisfied. Hence, condition (ii) is equivalent to Proposition 9(ii).

(iii) Given $x_{1}:=\left(u_{1}, v_{1}\right), x_{2}:=\left(u_{2}, v_{2}\right)$ and $(x, y) \in X \times Y$, we have representation (20), and consequently,

$$
\begin{aligned}
d\left((x, y),\left(\Omega_{1}-x_{1}\right) \cap\left(\Omega_{2}-x_{2}\right)\right) & \\
= & \max \left\{d\left(x+u_{1}, F^{-1}\left(y+v_{1}-v_{2}\right)\right),\left\|y+v_{2}-\bar{y}\right\|\right\} .
\end{aligned}
$$

Thus, inequality

$$
\alpha d\left((x, y),\left(\Omega_{1}-x_{1}\right) \cap\left(\Omega_{2}-x_{2}\right)\right) \leq \max \left\{d\left((x, y), \Omega_{1}-x_{1}\right), d\left((x, y), \Omega_{2}-x_{2}\right)\right\}
$$

implies inequality (27), and the converse implication is true if $\alpha \leq 1$. Hence, condition (iii) is equivalent to Proposition 9(iii).

The conclusions follow from Proposition 9.

Next we apply the metric estimates in Theorem 4 to establish relations between regularity properties of set-valued mappings in Definition 2 and the corresponding transversality properties of the collection of two sets (16).

Theorem 5 Let $X$ and $Y$ be normed spaces, $F: X \rightrightarrows Y,(\bar{x}, \bar{y}) \in \operatorname{gph} F, \Omega_{1}$ and $\Omega_{2}$ be defined by (16), $\alpha>0, \alpha_{1}:=\left(2 \alpha^{-1}+1\right)^{-1}$ and $\alpha_{2}:=\alpha / 2$.

(i) If $F$ is $\alpha$-semiregular at $(\bar{x}, \bar{y})$ with some $\delta>0$, then $\left\{\Omega_{1}, \Omega_{2}\right\}$ is $\alpha_{1}$-semitransversal at $(\bar{x}, \bar{y})$ with $\delta^{\prime}:=(1+\alpha / 2) \delta$.

Conversely, $F$ is $\alpha$-semiregular at $(\bar{x}, \bar{y})$ with some $\delta>0$ if $\left\{\Omega_{1}, \Omega_{2}\right\}$ is $\alpha_{2}$-semitransversal at $(\bar{x}, \bar{y})$ with $\delta$.

(ii) If $F$ is $\alpha$-subregular at $(\bar{x}, \bar{y})$ with some $\delta_{1}>0$ and $\delta_{2}>0$, then $\left\{\Omega_{1}, \Omega_{2}\right\}$ is $\alpha_{1}-$ subtransversal at $(\bar{x}, \bar{y})$ with any $\delta_{1}^{\prime}>0$ and $\delta_{2}^{\prime}>0$ satisfying $\delta_{1}^{\prime} \leq(1+\alpha / 2) \delta_{1}$ and $\alpha_{1} \delta_{1}^{\prime}+\delta_{2}^{\prime} \leq \delta_{2}$.

Conversely, $F$ is $\alpha$-subregular at $(\bar{x}, \bar{y})$ with some $\delta_{1}>0$ and $\delta_{2}>0$ if $\left\{\Omega_{1}, \Omega_{2}\right\}$ is $\alpha_{2}$-subtransversal at $(\bar{x}, \bar{y})$ with $\delta_{1}$ and $\delta_{2}^{\prime}:=\max \left\{\alpha_{2} \delta_{1}, \delta_{2}\right\}$.

(iii) If $F$ is $\alpha$-regular at $(\bar{x}, \bar{y})$ with some $\delta_{1}>0$ and $\delta_{2}>0$, then $\left\{\Omega_{1}, \Omega_{2}\right\}$ is $\alpha_{1}$-transversal at $(\bar{x}, \bar{y})$ with any $\delta_{1}^{\prime}>0$ and $\delta_{2}^{\prime}>0$ satisfying $\delta_{1}^{\prime} \leq(1+\alpha / 2) \delta_{1}$ and $\alpha_{1} \delta_{1}^{\prime}+\delta_{2}^{\prime} \leq \delta_{2} / 2$.

Conversely, $F$ is $\alpha$-regular at $(\bar{x}, \bar{y})$ with some $\delta_{1}>0$ and $\delta_{2}>0$ if $\left\{\Omega_{1}, \Omega_{2}\right\}$ is $\alpha_{2}$-transversal at $(\bar{x}, \bar{y})$ with $\delta_{1}$ and $\delta_{2}^{\prime}:=\alpha \delta_{1}+\delta_{2}$.

Proof Note that $\left.\alpha_{1} \in\right] 0,1\left[\right.$ and $\alpha_{1}<\alpha_{2}$.

(i) Let $F$ be $\alpha$-semiregular at $(\bar{x}, \bar{y})$ with some $\delta>0$. Set $\delta^{\prime}:=(1+\alpha / 2) \delta$. Let $u \in\left(\alpha_{1} \delta^{\prime}\right) \mathbb{B}_{X}$ and $v \in\left(2 \alpha_{1} \delta^{\prime}\right) \mathbb{B}_{Y}$. Observe that

$$
2 \alpha_{1} \delta^{\prime}=\frac{2(1+\alpha / 2)}{2 \alpha^{-1}+1} \delta=\alpha \delta
$$


Thus, $v \in(\alpha \delta) \mathbb{B}_{Y}$. In view of Definition 2(i),

$$
\begin{aligned}
d\left(\bar{x}+u, F^{-1}(\bar{y}+v)\right) & \leq d\left(\bar{x}, F^{-1}(\bar{y}+v)\right)+\|u\| \leq \alpha^{-1}\|v\|+\|u\| \\
& \leq\left(2 \alpha^{-1}+1\right) \max \left\{\|u\|, \frac{1}{2}\|v\|\right\}=\alpha_{1}^{-1} \max \left\{\|u\|, \frac{1}{2}\|v\|\right\} .
\end{aligned}
$$

By Theorem 4, $\left\{\Omega_{1}, \Omega_{2}\right\}$ is $\alpha_{1}$-semitransversal at $(\bar{x}, \bar{y})$ with $\delta^{\prime}$.

Conversely, let $\left\{\Omega_{1}, \Omega_{2}\right\}$ be $\alpha_{2}$-semitransversal at $(\bar{x}, \bar{y})$ with some $\delta>0$. Let $v \in(\alpha \delta) \mathbb{B}_{Y}$. Thus, $v \in\left(2 \alpha_{2} \delta\right) \mathbb{B}_{Y}$. By Theorem 4(i), $\alpha_{2} d\left(\bar{x}, F^{-1}(\bar{y}+v)\right) \leq\|v\| / 2$, and consequently, $\alpha d\left(\bar{x}, F^{-1}(\bar{y}+v)\right) \leq\|v\|$. Hence, by Definition 2(i), $F$ is $\alpha$-semiregular at $(\bar{x}, \bar{y})$ with $\delta$.

(ii) Let $F$ be $\alpha$-subregular at $(\bar{x}, \bar{y})$ with some $\delta_{1}>0$ and $\delta_{2}>0$. Choose $\delta_{1}^{\prime}>0$ and $\delta_{2}^{\prime}>0$ such that $\delta_{1}^{\prime} \leq(1+\alpha / 2) \delta_{1}$ and $\delta_{2}^{\prime}+\alpha_{1} \delta_{1}^{\prime} \leq \delta_{2}$. Observe that

$$
2 \alpha_{1} \delta_{1}^{\prime} \leq \frac{2(1+\alpha / 2)}{2 \alpha^{-1}+1} \delta_{1}=\alpha \delta_{1}
$$

Let $x \in B_{\delta_{2}^{\prime}}(\bar{x}), y \in B_{\min \left\{\alpha_{1} \delta_{1}^{\prime}, \delta_{2}^{\prime}\right\}}(\bar{y})$ with $d((x, y), \operatorname{gph} F)<\alpha_{1} \delta_{1}^{\prime}$. Then, for any number $\varepsilon$ with $d((x, y), \operatorname{gph} F)<\varepsilon<\alpha_{1} \delta_{1}^{\prime}$, there exists a point $\left(x^{\prime}, y^{\prime}\right) \in \operatorname{gph} F$ such that $\left\|(x, y)-\left(x^{\prime}, y^{\prime}\right)\right\|<\varepsilon$. Thus,

$$
\begin{gathered}
\left\|x^{\prime}-\bar{x}\right\| \leq\|x-\bar{x}\|+\left\|x^{\prime}-x\right\|<\delta_{2}^{\prime}+\alpha_{1} \delta_{1}^{\prime} \leq \delta_{2}, \\
d\left(\bar{y}, F\left(x^{\prime}\right)\right) \leq\left\|y^{\prime}-\bar{y}\right\| \leq\|y-\bar{y}\|+\left\|y^{\prime}-y\right\|<2 \alpha_{1} \delta_{1}^{\prime} \leq \alpha \delta_{1},
\end{gathered}
$$

and, in view of Definition 2(ii),

$$
\begin{aligned}
d\left(x, F^{-1}(\bar{y})\right) & \leq d\left(x^{\prime}, F^{-1}(\bar{y})\right)+\left\|x^{\prime}-x\right\| \\
& \leq \alpha^{-1} d\left(\bar{y}, F\left(x^{\prime}\right)\right)+\left\|x^{\prime}-x\right\| \\
& \leq \alpha^{-1}\left(\|y-\bar{y}\|+\left\|y^{\prime}-y\right\|\right)+\left\|x^{\prime}-x\right\| \\
& <\alpha^{-1}\|y-\bar{y}\|+\left(\alpha^{-1}+1\right) \varepsilon \\
& \leq\left(2 \alpha^{-1}+1\right) \max \{\|y-\bar{y}\|, \varepsilon\}=\alpha_{1}^{-1} \max \{\|y-\bar{y}\|, \varepsilon\} .
\end{aligned}
$$

Letting $\varepsilon \downarrow d((x, y), \operatorname{gph} F)$, we arrive at (18). By Theorem $4,\left\{\Omega_{1}, \Omega_{2}\right\}$ is $\alpha_{1}$-subtransversal at $(\bar{x}, \bar{y})$ with $\delta_{1}^{\prime}$ and $\delta_{2}^{\prime}$.

Conversely, let $\delta_{1}>0$ and $\delta_{2}>0$, and $\left\{\Omega_{1}, \Omega_{2}\right\}$ be $\alpha_{2}$-subtransversal at $(\bar{x}, \bar{y})$ with $\delta_{1}$ and $\delta_{2}^{\prime}:=\max \left\{\alpha_{2} \delta_{1}, \delta_{2}\right\}$. Let $x \in B_{\delta_{2}}(\bar{x})$ and $d(\bar{y}, F(x))<\alpha \delta_{1}$. Then, for any number $\varepsilon$ with $d(\bar{y}, F(x))<\varepsilon<\alpha \delta_{1}$, there exists a point $y^{\prime} \in F(x)$ such that $\left\|y^{\prime}-\bar{y}\right\|<\varepsilon$. Set $y:=\frac{y^{\prime}+\bar{y}}{2}$. Observe that

$$
\begin{gathered}
\left\|y-y^{\prime}\right\|=\|y-\bar{y}\|=\frac{\left\|y^{\prime}-\bar{y}\right\|}{2}<\frac{\alpha \delta_{1}}{2}=\alpha_{2} \delta_{1}, \\
\|y-\bar{y}\|<\delta_{2}^{\prime}, \quad d((x, y), \operatorname{gph} F) \leq\left\|y-y^{\prime}\right\|<\alpha_{2} \delta_{1} .
\end{gathered}
$$

By Theorem 4(ii),

$$
\alpha_{2} d\left(x, F^{-1}(\bar{y})\right) \leq \max \{d((x, y), \operatorname{gph} F),\|y-\bar{y}\|\}=\|y-\bar{y}\|=\frac{\left\|y^{\prime}-\bar{y}\right\|}{2}<\frac{\varepsilon}{2} .
$$

Thus, $\alpha d\left(x, F^{-1}(\bar{y})\right)<\varepsilon$. Letting $\varepsilon \downarrow d(\bar{y}, F(x))$, we obtain

$$
\alpha d\left(x, F^{-1}(\bar{y})\right) \leq d(\bar{y}, F(x))
$$

In view of Definition 2(ii), $F$ is $\alpha$-subregular at $(\bar{x}, \bar{y})$ with $\delta_{1}$ and $\delta_{2}$. 
(iii) Let $F$ be $\alpha$-regular at $(\bar{x}, \bar{y})$ with some $\delta_{1}>0$ and $\delta_{2}>0$. Choose $\delta_{1}^{\prime}>0$ and $\delta_{2}^{\prime}>0$ such that $\delta_{1}^{\prime} \leq(1+\alpha / 2) \delta_{1}$ and $\delta_{2}^{\prime}+\alpha_{1} \delta_{1}^{\prime} \leq \delta_{2} / 2$. Then we have (29). Let $(x, y) \in \operatorname{gph} F \cap B_{\delta_{2}^{\prime}}(\bar{x}, \bar{y}), u \in\left(\alpha_{1} \delta_{1}^{\prime}\right) \mathbb{B}_{X}$ and $v \in\left(2 \alpha_{1} \delta_{1}^{\prime}\right) \mathbb{B}_{Y}$. Set $y^{\prime}:=y+v$. Then

$$
\begin{gathered}
\|x-\bar{x}\|+\left\|y^{\prime}-\bar{y}\right\| \leq\|x-\bar{x}\|+\|y-\bar{y}\|+\|v\|<2 \delta_{2}^{\prime}+2 \alpha_{1} \delta_{1}^{\prime} \leq \delta_{2}, \\
d\left(y^{\prime}, F(x)\right) \leq\left\|y^{\prime}-y\right\|=\|v\|<2 \alpha_{1} \delta_{1}^{\prime} \leq \alpha \delta_{1}
\end{gathered}
$$

and, in view of Definition 2(iii),

$$
\begin{aligned}
d\left(x+u, F^{-1}(y+v)\right) & \leq d\left(x, F^{-1}\left(y^{\prime}\right)\right)+\|u\| \leq \alpha^{-1} d\left(y^{\prime}, F(x)\right)+\|u\| \\
& \leq \alpha^{-1}\|v\|+\|u\| \leq\left(2 \alpha^{-1}+1\right) \max \left\{\|u\|, \frac{1}{2}\|v\|\right\} \\
& =\alpha_{1}^{-1} \max \left\{\|u\|, \frac{1}{2}\|v\|\right\} .
\end{aligned}
$$

By Theorem 4, $\left\{\Omega_{1}, \Omega_{2}\right\}$ is $\alpha_{1}$-transversal at $(\bar{x}, \bar{y})$ with $\delta_{1}^{\prime}$ and $\delta_{2}^{\prime}$.

Conversely, let $\delta_{1}>0$ and $\delta_{2}>0$, and $\left\{\Omega_{1}, \Omega_{2}\right\}$ be $\alpha_{2}$-transversal at $(\bar{x}, \bar{y})$ with $\delta_{1}$ and $\delta_{2}^{\prime}:=\alpha \delta_{1}+\delta_{2}$. Let $x \in X$ and $y \in Y$ be such that $\|x-\bar{x}\|+\|y-\bar{y}\|<\delta_{2}$ and $d(y, F(x))<\alpha \delta_{1}$. Then, for any number $\varepsilon$ with $d(y, F(x))<\varepsilon<\alpha \delta_{1}$, there exists a point $y^{\prime} \in F(x)$ such that $\left\|y-y^{\prime}\right\|<\varepsilon$. Thus, $\left(x, y^{\prime}\right) \in \operatorname{gph} F$,

$$
\begin{aligned}
& \|x-\bar{x}\|<\delta_{2}<\delta_{2}^{\prime}, \quad\left\|y-y^{\prime}\right\|<\alpha \delta_{1}=2 \alpha_{2} \delta_{1}, \\
& \left\|y^{\prime}-\bar{y}\right\| \leq\left\|y^{\prime}-y\right\|+\|y-\bar{y}\|<\alpha \delta_{1}+\delta_{2}=\delta_{2}^{\prime} .
\end{aligned}
$$

By Theorem 4(iii),

$$
\alpha_{2} d\left(x, F^{-1}(y)\right) \leq \frac{\left\|y-y^{\prime}\right\|}{2}<\frac{\varepsilon}{2} .
$$

Thus, $\alpha d\left(x, F^{-1}(y)\right)<\varepsilon$. Letting $\varepsilon \downarrow d(y, F(x))$, we obtain $\alpha d\left(x, F^{-1}(y)\right) \leq$ $d(y, F(x))$. By Definition 2(iii), $F$ is $\alpha$-regular at $(\bar{x}, \bar{y})$ with $\delta_{1}$ and $\delta_{2}$.

The next corollary collects ' $\delta$-free' versions of the relations in Theorem 5 . It recaptures [29, Theorem 5.1].

Corollary 2 Let $X$ and $Y$ be normed spaces, $F: X \rightrightarrows Y,(\bar{x}, \bar{y}) \in \operatorname{gph} F$, and $\Omega_{1}, \Omega_{2}$ be defined by (16).

(i) $F$ is semiregular at $(\bar{x}, \bar{y})$ if and only if $\left\{\Omega_{1}, \Omega_{2}\right\}$ is semitransversal at $(\bar{x}, \bar{y})$. Moreover,

$$
\frac{1}{2 \mathrm{~s}_{\mathrm{e}} \operatorname{rg}[F](\bar{x}, \bar{y})^{-1}+1} \leq \mathrm{se}_{\mathrm{e}} \operatorname{tr}\left[\Omega_{1}, \Omega_{2}\right](\bar{x}) \leq \frac{\mathrm{s}_{\mathrm{e}} \operatorname{rg}[F](\bar{x}, \bar{y})}{2} .
$$

(ii) $F$ is subregular at $(\bar{x}, \bar{y})$ if and only if $\left\{\Omega_{1}, \Omega_{2}\right\}$ is subtransversal at $(\bar{x}, \bar{y})$. Moreover,

$$
\frac{1}{2 \operatorname{srg}[F](\bar{x}, \bar{y})^{-1}+1} \leq \operatorname{str}\left[\Omega_{1}, \Omega_{2}\right](\bar{x}) \leq \frac{\operatorname{srg}[F](\bar{x}, \bar{y})}{2}
$$

(iii) $F$ is regular at $(\bar{x}, \bar{y})$ if and only if $\left\{\Omega_{1}, \Omega_{2}\right\}$ is transversal at $(\bar{x}, \bar{y})$. Moreover,

$$
\frac{1}{2 \operatorname{rg}[F](\bar{x}, \bar{y})^{-1}+1} \leq \operatorname{tr}\left[\Omega_{1}, \Omega_{2}\right](\bar{x}) \leq \frac{\operatorname{rg}[F](\bar{x}, \bar{y})}{2} .
$$

Acknowledgements The authors thank the referees for the careful reading of the manuscript and their constructive comments and suggestions. We are also grateful to Guest Editor Prof Michel Théra for organizing the refereeing process perfectly.

The research was supported by the Australian Research Council, project DP160100854. The first author is supported by an Australian Government Research Training Program (RTP) Stipend and RTP Fee-Offset Scholarship through Federation University Australia. The third author benefited from the support of the FMJH Program PGMO and Conicyt REDES program 180032. 


\section{References}

1. Aragón Artacho, F.J., Mordukhovich, B.S.: Enhanced metric regularity and Lipschitzian properties of variational systems. J. Global Optim. 50(1), 145-167 (2011). DOI 10.1007/s10898-011-9698-x

2. Attouch, H., Bolte, J., Redont, P., Soubeyran, A.: Proximal alternating minimization and projection methods for nonconvex problems: an approach based on the Kurdyka-Łojasiewicz inequality. Math. Oper. Res. 35(2), 438-457 (2010). DOI 10.1287/moor.1100.0449

3. Bakan, A., Deutsch, F., Li, W.: Strong CHIP, normality, and linear regularity of convex sets. Trans. Amer. Math. Soc. 357(10), 3831-3863 (2005). DOI 10.1090/S0002-9947-05-03945-0

4. Bauschke, H.H., Borwein, J.M.: On projection algorithms for solving convex feasibility problems. SIAM Rev. 38(3), 367-426 (1996). DOI 10.1137/S0036144593251710

5. Bauschke, H.H., Borwein, J.M., Li, W.: Strong conical hull intersection property, bounded linear regularity, Jameson's property $(G)$, and error bounds in convex optimization. Math. Program., Ser. A 86(1), 135-160 (1999). DOI 10.1007/s101070050083

6. Bauschke, H.H., Borwein, J.M., Tseng, P.: Bounded linear regularity, strong CHIP, and CHIP are distinct properties. J. Convex Anal. 7(2), 395-412 (2000)

7. Bui, H.T., Kruger, A.Y.: Extremality, stationarity and generalized separation of collections of sets. J. Optim. Theory Appl. 182(1), 211-264 (2019). DOI 10.1007/s10957-018-01458-8

8. Cibulka, R., Fabian, M., Kruger, A.Y.: On semiregularity of mappings. J. Math. Anal. Appl. 473(2), 811-836 (2019). DOI 10.1016/j.jmaa.2018.12.071

9. Cuong, N.D., Kruger, A.Y.: Nonlinear semitransversality and transversality of collections of sets: Dual space characterizations. Preprint (2019)

10. Cuong, N.D., Kruger, A.Y.: Nonlinear subtransversality of collections of sets: Dual space characterizations. Preprint (2019)

11. Cuong, N.D., Kruger, A.Y.: Nonlinear transversality of collections of sets: Dual space sufficient characterizations. Preprint, Optimization Online: 2019-07-7313 (2019)

12. Cuong, N.D., Kruger, A.Y.: Nonlinear transversality of collections of sets: Primal space characterizations. Preprint, arXiv: 1902.06186 (2019)

13. Cuong, N.D., Kruger, A.Y.: Nonlinear transversality of collections of sets: Dual space necessary characterizations. J. Convex Anal. 27 (2020)

14. Dontchev, A.L., Rockafellar, R.T.: Implicit Functions and Solution Mappings. A View from Variational Analysis, 2 edn. Springer Series in Operations Research and Financial Engineering. Springer, New York (2014). DOI 10.1007/978-1-4939-1037-3

15. Drusvyatskiy, D., Ioffe, A.D., Lewis, A.S.: Transversality and alternating projections for nonconvex sets. Found. Comput. Math. 15(6), 1637-1651 (2015). DOI 10.1007/s10208-015-9279-3

16. Ioffe, A.D.: Metric regularity and subdifferential calculus. Russian Math. Surveys 55, 501-558 (2000). DOI 10.1070/rm2000v055n03ABEH000292

17. Ioffe, A.D.: Variational Analysis of Regular Mappings. Theory and Applications. Springer Monographs in Mathematics. Springer (2017). DOI 10.1007/978-3-319-64277-2

18. Klatte, D., Kummer, B.: Nonsmooth Equations in Optimization. Regularity, Calculus, Methods and Applications, Nonconvex Optimization and its Applications, vol. 60. Kluwer Academic Publishers, Dordrecht (2002)

19. Kruger, A.Y.: Stationarity and regularity of set systems. Pac. J. Optim. 1(1), 101-126 (2005)

20. Kruger, A.Y.: About regularity of collections of sets. Set-Valued Anal. 14(2), 187-206 (2006). DOI 10.1007/s11228-006-0014-8

21. Kruger, A.Y.: About stationarity and regularity in variational analysis. Taiwanese J. Math. 13(6A), 1737-1785 (2009). DOI 10.11650/twjm/1500405612

22. Kruger, A.Y.: About intrinsic transversality of pairs of sets. Set-Valued Var. Anal. 26(1), 111-142 (2018). DOI 10.1007/s11228-017-0446-3

23. Kruger, A.Y., López, M.A.: Stationarity and regularity of infinite collections of sets. J. Optim. Theory Appl. 154(2), 339-369 (2012). DOI 10.1007/s10957-012-0043-4

24. Kruger, A.Y., López, M.A.: Stationarity and regularity of infinite collections of sets. Applications to infinitely constrained optimization. J. Optim. Theory Appl. 155(2), 390-416 (2012). DOI 10.1007/ s10957-012-0086-6

25. Kruger, A.Y., Luke, D.R., Thao, N.H.: About subtransversality of collections of sets. Set-Valued Var. Anal. 25(4), 701-729 (2017). DOI 10.1007/s11228-017-0436-5

26. Kruger, A.Y., Luke, D.R., Thao, N.H.: Set regularities and feasibility problems. Math. Program., Ser. B 168(1-2), 279-311 (2018). DOI 10.1007/s10107-016-1039-x

27. Kruger, A.Y., Thao, N.H.: About uniform regularity of collections of sets. Serdica Math. J. 39(3-4), 287-312 (2013)

28. Kruger, A.Y., Thao, N.H.: About $[q]$-regularity properties of collections of sets. J. Math. Anal. Appl. 416(2), 471-496 (2014). DOI 10.1016/j.jmaa.2014.02.028

29. Kruger, A.Y., Thao, N.H.: Quantitative characterizations of regularity properties of collections of sets. J. Optim. Theory Appl. 164(1), 41-67 (2015). DOI 10.1007/s10957-014-0556-0

30. Kruger, A.Y., Thao, N.H.: Regularity of collections of sets and convergence of inexact alternating projections. J. Convex Anal. 23(3), 823-847 (2016)

31. Lewis, A.S., Luke, D.R., Malick, J.: Local linear convergence for alternating and averaged nonconvex projections. Found. Comput. Math. 9(4), 485-513 (2009). DOI 10.1007/s10208-008-9036-y

32. Li, C., Ng, K.F., Pong, T.K.: The SECQ, linear regularity, and the strong CHIP for an infinite system of closed convex sets in normed linear spaces. SIAM J. Optim. 18(2), 643-665 (2007). DOI 10.1137/ 060652087 
33. Mordukhovich, B.S.: Variational Analysis and Generalized Differentiation. I: Basic Theory, Grundlehren der Mathematischen Wissenschaften [Fundamental Principles of Mathematical Sciences], vol. 330. Springer, Berlin (2006)

34. Ng, K.F., Yang, W.H.: Regularities and their relations to error bounds. Math. Program., Ser. A 99(3), 521-538 (2004). DOI 10.1007/s10107-003-0464-9

35. $\mathrm{Ng}$, K.F., Zang, R.: Linear regularity and $\phi$-regularity of nonconvex sets. J. Math. Anal. Appl. 328(1), 257-280 (2007). DOI 10.1016/j.jmaa.2006.05.028

36. Ngai, H.V., Théra, M.: Metric inequality, subdifferential calculus and applications. Set-Valued Anal. 9(1-2), 187-216 (2001). DOI 10.1023/A:1011291608129

37. Noll, D., Rondepierre, A.: On local convergence of the method of alternating projections. Found. Comput. Math. 16(2), 425-455 (2016). DOI 10.1007/s10208-015-9253-0

38. Penot, J.P.: Calculus Without Derivatives, Graduate Texts in Mathematics, vol. 266. Springer, New York (2013). DOI 10.1007/978-1-4614-4538-8

39. Rockafellar, R.T., Wets, R.J.B.: Variational Analysis. Springer, Berlin (1998)

40. Zheng, X.Y., Ng, K.F.: Linear regularity for a collection of subsmooth sets in Banach spaces. SIAM J. Optim. 19(1), 62-76 (2008). DOI 10.1137/060659132

41. Zheng, X.Y., Wei, Z., Yao, J.C.: Uniform subsmoothness and linear regularity for a collection of infinitely many closed sets. Nonlinear Anal. 73(2), 413-430 (2010). DOI 10.1016/j.na.2010.03.032 